\title{
Implementation of an Effective Bond Energy Formalism in the Multicomponent Calphad Approach
}

\author{
Nathalie Dupin ${ }^{1}$, Ursula R. Kattner ${ }^{2}$, Bo Sundman ${ }^{3}$, Mauro Palumbo ${ }^{4}$, and Suzana G. Fries ${ }^{5}$ \\ ${ }^{1}$ Calcul Thermodynamique, \\ 63670 Orcet, France \\ ${ }^{2}$ National Institute of Standards and Technology, \\ Gaithersburg, MD 20899, USA \\ ${ }^{3} \mathrm{KTH}$ Royal Institute of Technology, \\ 10044 Stockholm, Sweden \\ ${ }^{4}$ COMPUMAT, \\ 10095 Grugliasco (TO), Italy \\ ${ }^{5}$ Ruhr-University Bochum, \\ 44801 Bochum, Germany \\ nathdupin@wanadoo.fr \\ ursula.kattner@nist.gov \\ bo.sundman@gmail.com \\ mauropalumbo75@gmail.com \\ suzana.g.fries@ruhr-uni-bochum.de
}

Most models currently used for complex phases in the calculation of phase diagrams (Calphad) method are based on the compound energy formalism. The way this formalism is presently used, however, is prone to poor extrapolation behavior in higher-order systems, especially when treating phases with complex crystal structures. In this paper, a partition of the Gibbs energy into effective bond energies, without changing its configurational entropy expression, is proposed, thereby remarkably improving the extrapolation behavior. The proposed model allows the use of as many sublattices as there are occupied Wyckoff sites and has great potential for reducing the number of necessary parameters, thus allowing shorter computational time. Examples for face centered cubic (fcc) ordering and the $\sigma$ phase are given.

Key words: Calphad; compound energy formalism; effective bond energy formalism.

Accepted: October 22, 2018.

Published: November 26, 2018

https://doi.org/10.6028/jres.123.020

\section{Introduction}

The calculation of phase diagrams (Calphad) approach $[1,2]$ is a method used in many areas in order to describe the thermodynamic properties of materials. Phase diagram information is historically its main 
output but is far from being the only one. The method potentially allows the description of all the thermodynamic properties, including enthalpies of reaction, heat capacities, activities, partial pressures, and driving forces or thermodynamic factors, at stable or metastable equilibrium.

Most of the Calphad descriptions are based on assessments of simple systems, namely, binary and ternary systems. The strength of this approach is its ability to extrapolate to higher-order systems, e.g., quaternary, with a high level of accuracy. This is possible because of the flexibility and the simplicity of the compound energy formalism (CEF), which is the basis for most of the descriptions. The CEF is based on the existence of different sublattices in the crystal structure of many phases. For the sake of clarity, the CEF is presented in the first part of this contribution, and its application to the $L 1_{2}\left(\gamma^{\prime}\right)$ and $\sigma$ phases is discussed. The ternary extrapolation of the $\sigma$ phase using only binary parameters compared to a calculation using binary and ternary parameters revealed limitations of the current CEF, leading to the introduction of a new implementation of this formalism using effective bond energies between the elements as model parameters. Examples of the use of this effective bond energy formalism (EBEF), already implemented in the OpenCalphad (OC) software [3], are given.

\section{Compound Energy Formalism}

The CEF $[4,5]$ is a very flexible formalism allowing the description of many different kinds of phases. In order to present this formalism, a hypothetical phase, $\varphi$, will be considered. While this general formalism is often presented using a two-sublattice (2SL) model, we will consider a more complex case with four sublattices (4SL) in order to use this example also for the introduction of the new formalism, which provides no benefit for a $2 \mathrm{SL}$ model. Consider the following model:

$$
(\mathrm{A}, \mathrm{B}, \ldots)_{a_{1}^{\varphi}}(\mathrm{A}, \mathrm{B}, \ldots)_{a_{2}^{\varphi}}(\mathrm{A}, \mathrm{B}, \ldots)_{a_{3}^{\varphi}}(\mathrm{A}, \mathrm{B}, \ldots)_{a_{4}^{\varphi}}
$$

where $\mathrm{A}, \mathrm{B}, \ldots$, are elements, and $a_{1}^{\varphi}, a_{2}^{\varphi}, a_{3}^{\varphi}$, and $a_{4}^{\varphi}$ are the number of sites corresponding to the different sublattices, i.e., the $1^{\text {st }}, 2^{\text {nd }}, 3^{\text {rd }}$, and $4^{\text {th }}$ sublattice, respectively. Although they can actually be taken as the multiplicities of the Wyckoff sites corresponding to the sublattices of the crystallographic structure, this is not imposed by the formalism, and many phases have been modeled with this formalism using simplified sublattice models. The total number of sites defined by this model is $n_{s}^{\varphi}=\sum_{s} a_{s}^{\varphi}$.

The fraction of the element $i$ in the $n^{\text {th }}$ sublattice is $y_{i}^{n}$. Therefore, $y_{\mathrm{A}}^{1}$ stands for the fraction of the $1^{\text {st }}$ sublattice occupied by A. The sum of fractions of all the elements in a given sublattice equals unity: $\sum_{i} y_{i}^{s}=1$. The atomic fraction of the element $i$ in the phase $\varphi$ is thus $x_{i}^{\varphi}=\sum_{s} a_{s}^{\varphi} y_{i}^{s} / n_{s}^{\varphi}$.

\subsection{Expression of the Gibbs Energy}

The Gibbs energy for this phase, $\varphi$, is expressed in the CEF as:

$$
G^{\varphi}-n_{s}^{\varphi} \sum_{i} x_{i}^{\varphi} H_{i}^{\mathrm{SER}}={ }^{\mathrm{rf}} \Delta^{\circ} G^{\varphi}+R T \sum_{s} a_{s}^{\varphi} \sum_{i} y_{i}^{s} \ln y_{i}^{s}+{ }^{\mathrm{xs}} G^{\varphi}
$$

and is defined relative to the enthalpy at $298.15 \mathrm{~K}$ of the elements in their reference state, $H_{i}^{\mathrm{SER}}$. This reference is common to all the phases considered in a multicomponent database in order to be able to compute phase equilibria between the different competing phases. Constraints on the variables $x_{i}^{\varphi}$ and $y_{i}^{s}$, described above, are needed to minimize this function.

The first term on the right side in Eq. (1) is a reference term describing the mechanical mixing of all the stoichiometric compounds defined by the model. These are also called end members, because they represent limiting compositions with no configurational entropy. 


$$
\begin{gathered}
{ }^{\mathrm{rf}} \Delta^{\circ} G^{\varphi}=\sum_{i} \sum_{j} \sum_{k} \sum_{l} y_{i}^{1} y_{j}^{2} y_{k}^{3} y_{l}^{4} \Delta^{\circ} G_{i: j: k: l}^{\varphi} \\
\Delta^{\circ} G_{i: j: k: l}^{\varphi}=G_{i: j: k: l}^{\varphi}-a_{1}^{\varphi} H_{i}^{\mathrm{SER}}-a_{2}^{\varphi} H_{j}^{\mathrm{SER}}-a_{3}^{\varphi} H_{k}^{\mathrm{SER}}-a_{4}^{\varphi} H_{l}^{\mathrm{SER}}
\end{gathered}
$$

$G_{i: j: k: l}^{\varphi}$ is the Gibbs energy of the end member $i_{a_{1}^{\varphi}} j_{a_{2}^{\varphi}} k_{a_{3}^{\varphi}} l_{a_{4}^{\varphi}}$. Colons separate the elements that are in different sublattices. $\Delta^{\circ} G_{i: j: k: l}^{\varphi}$ is the Gibbs energy of this compound referred to the reference states of the elements.

When the same element occupies different sublattices $(i: i: i: i)$, the compound under consideration corresponds to the pure element $i$ in the structure $\varphi$. Depending on the phase, this state can be stable, metastable, or hypothetical (i.e., unstable) for the element. When considering the $\sigma$ phase, most of the pure elements are in a hypothetical state. When considering the $L 1_{2}$ phase, the pure elements correspond to the face centered cubic (fcc) $A 1$ structure, which can be a stable state.

The second term on the right side of Eq. (1) is the contribution from the configurational entropy to the Gibbs energy, assuming random mixing of all species in each sublattice. The third term, ${ }^{\mathrm{xs}} G^{\varphi}$, is the excess term, where the different parameters represent the interaction of species in the same sublattice. However, details of the description of the excess term will not be discussed here, because they can be found in Ref. [2].

\subsection{The $\sigma$ Phase}

The $\sigma$ phase appears in many different types of engineering alloys. The methods used to control its formation during processing require a reliable thermodynamic description, which is thus of high interest. This phase is stable in many binary systems, with widely varying composition ranges of stability and widely varying degrees of chemical order. The stable $\sigma$ phases in binary systems extend their stability field into many ternary systems following different topologies. Stable $\sigma$ phases can also be found in ternary systems where they are not stable in the constituting binary systems. This feature, as well as the stabilization of binary phases at higher temperature when they are not stable at lower temperature, could be explained by the contribution of the configurational entropy.

Table 1. Crystallographic data of the $\sigma$ phase.

\begin{tabular}{lccccc}
\hline Prototype & $\mathrm{CrFe}$ & & & \\
\hline Strukturbericht & \multicolumn{2}{l}{$D 8_{b}$} & & & \\
\hline Space group & $P 4_{2} / m n m$ & & & \\
\hline Pearson Symbol & $t P 30$ & & & & \\
\hline Wyckoff position & $2 \mathrm{a}$ & $4 \mathrm{f}$ & $8 \mathrm{i}_{1}$ & $8 \mathrm{i}_{2}$ & $8 \mathrm{j}$ \\
Point symmetry & $\mathrm{mmm}$ & $\mathrm{mm}$ & $\mathrm{m}$ & $\mathrm{m}$ & $\mathrm{m}$ \\
Coordination number & 12 & 15 & 14 & 12 & 14 \\
Fe \% in CrFe & 88 & 25 & 38 & 84 & 34 \\
\hline
\end{tabular}

The crystallographic structure of the $\sigma$ phase is summarized in Table 1. It shows five distinct sites. Different features of this phase and its description with the CEF have been discussed in the past $[6,7]$. They will be briefly summarized here and extended to the current state of the art, and the remaining difficulties in the application to multicomponent thermodynamic databases will be discussed. 
In the early application of the CEF, due to the limitation of computers at that time and the difficulty of assessing the Gibbs energy of formation of metastable end members, it was thought impossible to use a model to render the full complexity of its crystallographic structure. It was simplified to a 3SL model that can be schematized as $(\mathrm{A})_{a}(\mathrm{~A}, \mathrm{~B})_{c}(\mathrm{~B})_{b}$, distinguishing two kinds of atoms. The first sublattice is occupied by $\mathrm{A}$ atoms, the last sublattice is occupied by $\mathrm{B}$ atoms, and the second sublattice is occupied by a mixture of both kinds. The stable structures of the majority of the transition metals are face centered cubic (fcc), body centered cubic (bcc), and hexagonal close packed (hcp). The A atoms are defined as transition metals on the left of the periodic table, most with stable bcc structure. The B atoms are transition metals on the right of the periodic table, most with stable fcc structure. This $\sigma$ phase model followed the idea that sites with a coordination number of 12 ( $2 \mathrm{a}$ and $8 \mathrm{i}_{2}$ ) were mostly occupied by B atoms, the site with a coordination number of 15 (4f) was mostly occupied by A atoms, and the sites with an intermediate coordination number $\left(8 i_{1}\right.$ and $8 \mathrm{j}$ ) had a mixed occupancy. In a binary system of the A-B kind, such a model allows description of the composition range between $\mathrm{A}_{a+c} \mathrm{~B}_{b}$ and $\mathrm{A}_{a} \mathrm{~B}_{b+c}$.

However, when using the value from the crystallographic structure where $a=4, b=10$, and $c=16$, the model did not allow description of the homogeneity range of the $\sigma$ phase in some systems. The model was thus modified and used with values $a=4, b=8$, and $c=18$ [8]. The 4/8/18 model was applied to many systems until Ansara et al. [6] recommended the use of the site numbers reflecting the occupation of the crystallographic sites, i.e., 4/10/16, with the possibility of considering the presence of A atoms on the site previously reserved for B atoms in the systems where the model did not allow the proper description of the experimental information.

Ansara et al. [9] later introduced a new formalism that is closer to the crystallography of any topologically close packed (TCP) phase by allowing all elements in all the sublattices. Inspired by the cluster expansion approach [10], the new formalism, called the extended Calphad method, adds a contribution to the classical CEF that is a function of the composition of the phase, i.e., independent of the degree of ordering. The Gibbs energy is then expressed as:

$$
G^{\varphi}-n_{s}^{\varphi} \sum_{i} x_{i}^{\varphi} H_{i}^{\mathrm{SER}}=n_{s}^{\varphi} \Delta G^{\varphi}\left(x_{i}^{\varphi}\right)+\Delta G^{\varphi}\left(y_{i}^{s}\right)
$$

where $\Delta G^{\varphi}\left(x_{i}^{\varphi}\right)$ is a configuration-independent function of the composition of the phase similar to the formalism used for disordered phases but where the ideal entropy term is ignored. This term includes the reference terms for the enthalpy:

$$
\Delta G^{\varphi}\left(x_{i}^{\varphi}\right)=\sum_{i} x_{i}^{\varphi}\left(G_{i}^{\varphi}-H_{i}^{\mathrm{SER}}\right)+\sum_{i} \sum_{j>i} x_{i}^{\varphi} x_{j}^{\varphi} L_{i, j}^{\varphi}
$$

where $G_{i}^{\varphi}$ is the molar Gibbs energy of the pure element $i$ in the structure of the phase $\varphi$. The terms $L_{i, j}^{\varphi}$ are the so-called Redlich-Kister interaction parameters, which can depend on composition and temperature, and $\Delta G^{\varphi}\left(y_{i}^{S}\right)$ is a function of the site occupation of the sublattices similar to Eq. (1).

$$
\Delta G^{\varphi}\left(y_{i}^{s}\right)={ }^{\mathrm{rf}} \Delta G^{\varphi}+R T \sum_{s} a_{s}^{\varphi} \sum_{i} y_{i}^{s} \ln y_{i}^{s}+{ }^{\mathrm{xs}} G^{\varphi}
$$

The introduction of the composition-dependent term in Eq. (4) results in a change of reference state for the Gibbs energy of the end members in ${ }^{\mathrm{rf}} \Delta^{\circ} G^{\varphi}$. These are now relative to the Gibbs energy of the pure elements in the $\varphi$ state and not the general reference state:

$$
\Delta G_{i: j: k: l}^{\varphi}=G_{i: j: k: l}^{\varphi}-a_{1}^{\varphi} G_{i}^{\varphi}-a_{2}^{\varphi} G_{j}^{\varphi}-a_{3}^{\varphi} G_{k}^{\varphi}-a_{4}^{\varphi} G_{l}^{\varphi}
$$

where $\Delta G_{i: j: k: l}^{\varphi}$ corresponds to the formation Gibbs energy of the end member $i_{a_{1}^{\varphi}} j_{a_{2}^{\varphi}} k_{a_{3}^{\varphi}} l_{a_{4}^{\varphi}}$ from the pure elements in the $\varphi$ state. This formalism allows that values are not assigned for all of the end members, i.e., 
$\Delta G_{i: j: k: l}^{\varphi}=0$, with the vibrational contribution being included in the order-independent term, $G_{i}^{\varphi}-H_{i}^{\mathrm{SER}}$. This is an interesting feature for the management of big databases. This formalism has been applied to the $\sigma$ phase in newer versions of a commercial thermodynamic database for Ni-base alloys, TCNI [11, 12]. ${ }^{1}$ Details have been discussed by Hallstedt et al. [13] in the case of the ternary system Co-Cr-Re.

Even with this new formalism, the systematic simplification to 3SL was widely used to assess as few parameters as possible until the density functional theory (DFT) approach allowed evaluation of a greater number of parameters. Fries and Sundman [14], using the 32 energies of formation from DFT in the Re-W system in a 5SL CEF model, showed that the CEF was able to reproduce the experimental site occupations of the $\sigma$ phase with equal accuracy as the cluster variation method (CVM) approach [15], which uses a much more complicated entropy expression. They also showed that it was possible to assess the phase diagram based on this model.

Many studies of the $\sigma$ phase coupling DFT-calculated formation energies and the CEF have been published. However, only a few assessments have been carried out considering all the phases of the system to obtain a so-called Calphad description [14, 16-18]. Many studies used DFT inputs, keeping the old simplified models where a reduced composition range was considered. Mathieu et al. [17] showed that this resulted in quite unrealistic thermodynamic properties. At the composition corresponding to the limit of the model definition, the configuration is fixed and cannot reflect the correct enthalpy and the entropy when the temperature changes compared to the complete 5SL description.

Mathieu et al. [17] studied other simplifications. As discussed by Palumbo et al. [16] for the case of Cr-Re, the 2SL simplification proposed by Joubert [7] is a poor approximation. In the Mo-Re case, the 3SL model 10/12/8, considering all the elements in the three sublattices, was shown to be the best approximation. This simplified model is different from the general recommendations by Ansara et al. [6]. This is in agreement with the study of different systems by Korzhavyi et al. [19]: A single simplified model cannot properly describe the site occupation preferences of the $\sigma$ phase in all of the systems where the phase appears.

It appears to be important to use a 5SL model in order to properly describe the configuration of the $\sigma$ phase in multicomponent alloys. Since computer speed has significantly increased over the past decades, such improvements are now computationally affordable. The difficulty remains in assessing a large number of model parameters. In the framework of a thermodynamic database for alloy and process development, in order to describe the stability of the $\sigma$ phase considering 16 elements mixing on 5SL, more than a million end members must be considered.

DFT results have been shown to be important for the development of high-quality binary Calphad descriptions, but an extensive effort to model millions of compounds in all systems up to quinary ones is currently not conceivable. Even if the formalism presented in Eq. (4) allows consideration of only some of the configurations, guessing which configurations must be evaluated is not obvious and will depend on the system under consideration. The need to assess higher-order compounds when using complex CEF descriptions is illustrated by Fig. 1 and Fig. 2.

Figure 1 corresponds to the extrapolation of the binary descriptions [17, 20,21] into the ternary system Mo-Ni-Re using the extended CEF [9] for the $\sigma$ phase and mixing all of the elements in 5SL. In addition to the stable phase descriptions, all of the $\Delta G_{i: j: k: l: m}^{\sigma}$ values in Eq. (7) are set to DFT results [22] for all 90, i.e., $3 \times\left(2^{5}-2\right)$, of the binary compounds. The model moreover defines 150 ternary end members. ${ }^{2}$ No values are introduced for their Gibbs energy of formation; they are set equal to zero. The $\sigma$ description

${ }^{1}$ Certain commercial equipment, products, instruments, or materials are identified in this paper for reference. Such identification does not imply recommendation or endorsement by the National Institute of Standards and Technology, nor does it imply that the materials or equipment identified are necessarily the best available for the purpose.

${ }^{2}$ A 5SL model considering three elements defines $3^{5}$ end members, i.e., 243 configurations, of which three consist of only one element and 90 consist of only two elements, which leaves 150 ternary configurations. 


\section{Journal of Research of National Institute of Standards and Technology}

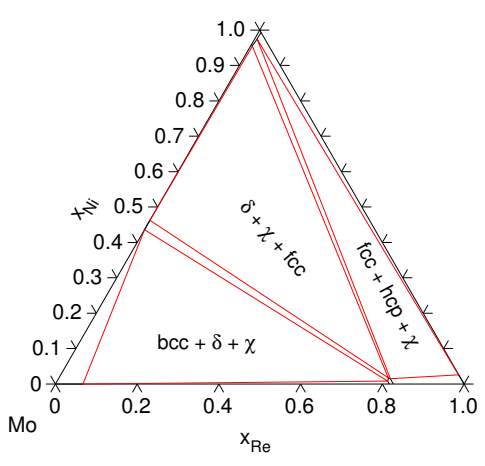

$\mathrm{T}=500 \mathrm{~K}$

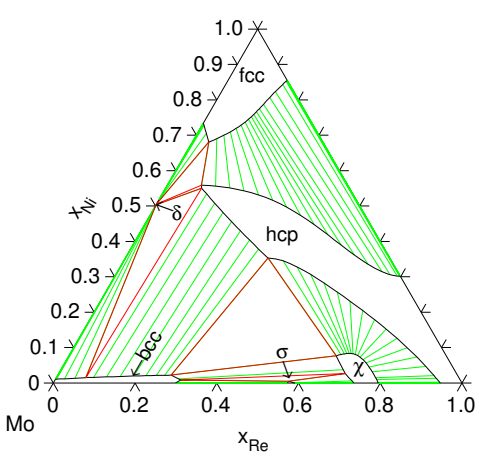

$\mathrm{T}=1500 \mathrm{~K}$

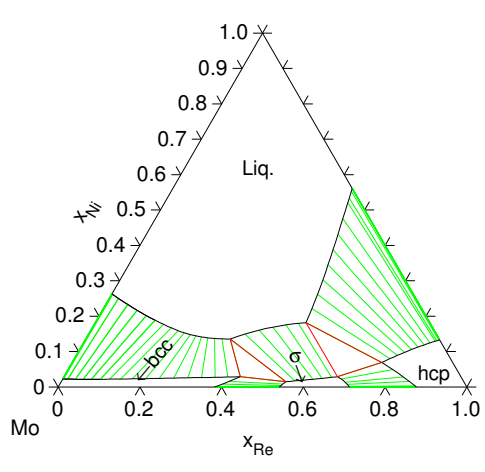

$\mathrm{T}=2500 \mathrm{~K}$

Fig. 1. Isothermal sections in the Mo-Ni-Re system calculated for $500 \mathrm{~K}, 1500 \mathrm{~K}$, and $2500 \mathrm{~K}$ with the CEF using the formation energies from DFT [22] for only the 90 , i.e., $3 \times\left(2^{5}-2\right)$, binary configurations. This $\sigma$ description is presented in Sec. 5.2.1.

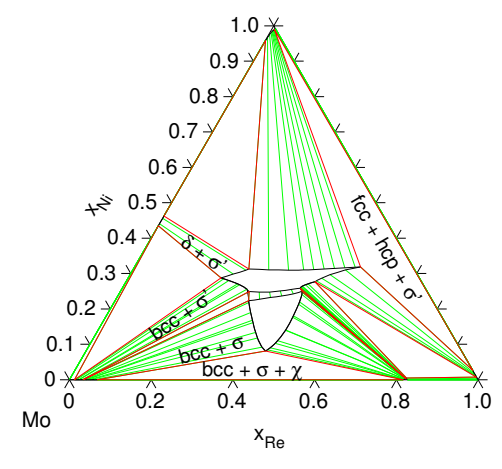

$\mathrm{T}=500 \mathrm{~K}$

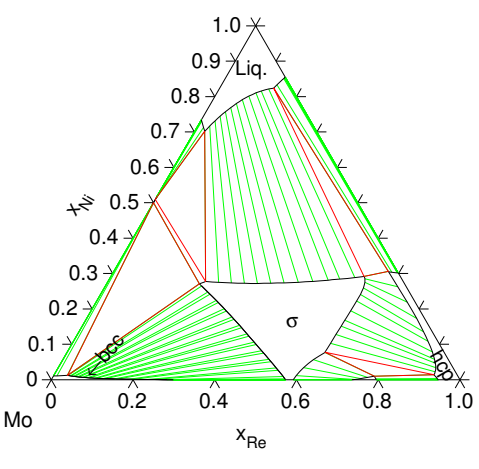

$\mathrm{T}=1500 \mathrm{~K}$

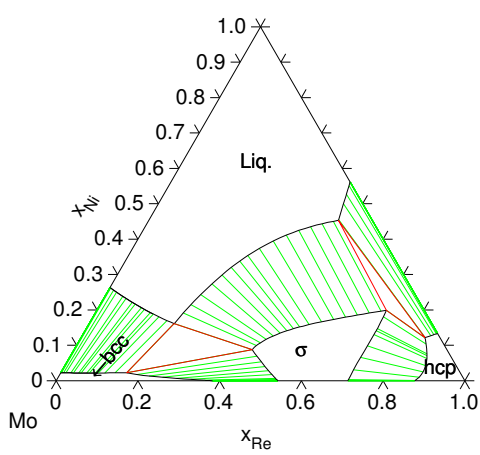

$\mathrm{T}=2500 \mathrm{~K}$

Fig. 2. Isothermal sections in the Mo-Ni-Re system calculated for $500 \mathrm{~K}, 1500 \mathrm{~K}$, and $2500 \mathrm{~K}$ with the CEF using binary and ternary compound formation energies (240, i.e., $3^{5}-3$, compounds) from DFT [22]. This $\sigma$ description is presented in Sec. 5.2.2.

corresponding to this case is presented in Sec. 5.2.1. The $\sigma$ phase is stable in the binary system Mo-Re at $1500 \mathrm{~K}$ and $2500 \mathrm{~K}$. Its extension in the ternary system is very small and in disagreement with the experimental data [23-27].

Figure 2 shows the isothermal sections at the same temperatures when the parameters of the 150 ternary compounds are also set to DFT results [22], i.e., using the $\sigma$ description presented in Sec. 5.2.2. The $\sigma$ phase largely extends into the ternary system. It is even stabilized in the ternary system at $500 \mathrm{~K}$, although it is not stable in the Mo-Re system at this temperature. No experimental information is available at this temperature to confirm this feature. The $\sigma$ stability field at $1500 \mathrm{~K}$ is similar to that reported experimentally in this temperature range. These sections do not constitute a proper Calphad assessment, but they demonstrate that input from DFT can make a significant difference.

The differences between Fig. 1 and Fig. 2 are the result of whether or not the 150 ternary parameters are set. They show the poor ability of the model to extrapolate when only binary compounds are assessed. A better way is needed to extrapolate the knowledge from the binary systems, where extensive DFT can be considered, towards higher-order systems, particularly those in which DFT is too expensive to comprehensively sample. 


\subsection{The $\gamma^{\prime}$ phase}

The $\gamma^{\prime}$ phase is simpler than the $\sigma$ phase from a crystallographic viewpoint. Understanding how the CEF allowed its description in the complex composition range of interest for superalloys helps to develop similar strategies for complex phases.

The $\gamma^{\prime}$ phase has the $L 1_{2}$ crystal structure shown in Fig. 3. It is an ordered variant of the fcc $(\gamma, A 1)$ phase, for which the crystal structure is also shown in Fig. 3. A good thermodynamic description of these two phases is very important because their simultaneous presence results in the high strength of some superalloys. Within the Calphad approach, different types of modeling of these phases have been reported for the Al-Ni prototype system. One of the most widely used models $[6,11,28,29]$ is based on the idea developed by Shockley [30] to describe these two phases as well as the $L 1_{0}$ ordering, also shown in Fig. 3 , with a single model using the bond exchange energy, referred to here as bond energy, between first neighbors of the fcc structure.We use $\varepsilon_{A B}$ to denote this bond energy between the elements $\mathrm{A}$ and $\mathrm{B}$.

a)

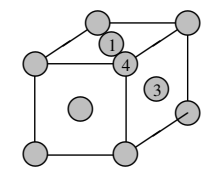

b)

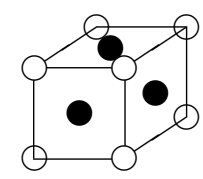

c)

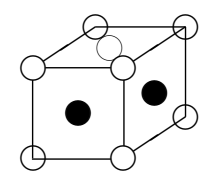

Fig. 3. Schematic cells of the $A 1$ (a), $L 1_{2}$ (b), and $L 1_{0}$ (c) structures.

In order to apply this model within the CEF, four equivalent SLs have to be considered. Each SL corresponds to a site labeled with an integer in Fig. 3a. The bond energy between A and B occupying the sublattices $s$ and $r$, respectively, is denoted $\varepsilon_{A B}^{s r}$. The symmetry of the structure implies that the bond energy between two of these sites is independent of the sites under consideration.

$$
\varepsilon_{A B}^{s r}=\varepsilon_{B A}^{s r}=\varepsilon_{A B}^{s t}=\cdots=\varepsilon_{A B}
$$

Adding the energies corresponding to the six bonds linking the four equivalent SLs, the Gibbs energy of formation of the end members can be written as:

$$
\Delta G_{i: j: k: l}^{\varphi}=\varepsilon_{i j}+\varepsilon_{i k}+\varepsilon_{i l}+\varepsilon_{j k}+\varepsilon_{j l}+\varepsilon_{k l}
$$

The references for this Gibbs energy of formation are the pure elements in the structure under consideration, and the energy of bonds between two identical elements is zero; $\varepsilon_{i i}=0$. The relation in Eq. (9) keeps the Shockley assumption of a single bond energy, independent of composition and temperature. Such an assumption is not necessary in the CEF or extended CEF, because each $\Delta G_{i: j: k: l}^{\varphi}$ can be set independently, especially for systems where more experimental or theoretical information is available. However, during the development of a commercial database for Ni-base alloys [11], it was shown that this assumption is important in order to allow reasonable extrapolations in many systems where few data are available. It can be considered as a first approximation, and more terms can be added, if needed, for a few end members. An example of such use can be found in the description of this phase in the ternary system Al-Cr-Ni [28]:

$$
\Delta G_{A l: C r: N i: N i}^{\varphi}=\varepsilon_{A l C r}+2 \varepsilon_{A l N i}+2 \varepsilon_{C r N i}+\varepsilon_{A l C r N i_{2}}
$$

The first three terms in Eq. (10) correspond to the approximation introduced by Eq. (9). An extra term is added to account for the fact that the bond energies depend on the surroundings. Calculations using the coherent potential approximation have shown that the addition of other elements to a binary phase may affect the pair interactions in this phase [31]. This indicates that bond energies in triplets or quadruplets may 
also have to be taken into account. Assessment of such an extra term for Al-Cr-Ni was possible because there is sufficient experimental information for this system available.

The use of relations such as Eq. (9) or Eq. (10) allows a significant reduction in the number of independent functions required to express the formation Gibbs energies of all of the end members. However, the fact that the $\varepsilon_{A B}$ parameters are not available in the CEF makes it mandatory to express the Gibbs energy of formation of all the end members with respect to these bond parameters. The simplification is thus only conceptual, and the resulting database is large and difficult to handle. The $\varepsilon_{A B}$ parameters will constitute the basis of the formalism introduced in the next section.

\section{The Effective Bond Energy Formalism}

The general form of Gibbs energy of a phase described with the EBEF proposed here is the same as for a phase described by the extended CEF in Eq. (4). However, the ordering part is now expanded as a function of different energies that characterize the different contributions to the configuration.

$$
\begin{aligned}
\Delta G^{\varphi}\left(y_{i}^{s}\right)= & \sum_{i} \sum_{j \neq i}\left(y_{i}^{1} y_{j}^{2} \Delta^{\circ} G_{i: j: *: *}^{\varphi}+y_{i}^{1} y_{j}^{3} \Delta^{\circ} G_{i: *: j: *}^{\varphi}+y_{i}^{1} y_{j}^{4} \Delta^{\circ} G_{i: *: *: j}^{\varphi}\right. \\
& \left.+y_{i}^{2} y_{j}^{3} \Delta^{\circ} G_{*: i: j: *}^{\varphi}+y_{i}^{2} y_{j}^{4} \Delta^{\circ} G_{*: i: *: j}^{\varphi}+y_{i}^{3} y_{j}^{4} \Delta^{\circ} G_{*: *: i: j}^{\varphi}\right) \\
& +\sum_{i} \sum_{j} \sum_{k}\left(y_{i}^{1} y_{j}^{2} y_{k}^{3} \Delta^{\circ} G_{i: j: k: *}^{\varphi}+y_{i}^{1} y_{j}^{2} y_{k}^{4} \Delta^{\circ} G_{i: j: *: k}^{\varphi}\right. \\
& \left.+y_{i}^{1} y_{j}^{3} y_{k}^{4} \Delta^{\circ} G_{i: *: j: k}^{\varphi}+y_{i}^{2} y_{j}^{3} y_{k}^{4} \Delta^{\circ} G_{*: i: j: k}^{\varphi}\right) \\
& +\sum_{i} \sum_{j} \sum_{k} \sum_{l} y_{i}^{1} y_{j}^{2} y_{k}^{3} y_{l}^{4} \Delta^{\circ} G_{i: j: k: l}^{\varphi} \\
& +R T \sum_{s} a_{s}^{\varphi} \sum_{i} y_{i}^{s} \ln y_{i}^{s}+{ }^{\mathrm{xs}} G^{\varphi}
\end{aligned}
$$

The first set of summations considers bonds between two kinds of elements. For a model considering $n$ SLs, there are $n(n-1) / 2$ pairs of sites to consider. A pair of sites allows two possible occupations by two elements A and B: A-B and B-A. There are thus $n(n-1)$ possible effective bond energies for each binary system. The parameter $\Delta^{\circ} G_{i: j: *: *}^{\varphi}$ is the effective bond energy between $i$ in the first sublattice and $j$ in the second sublattice. This energy is the contribution of this effective bond. It is independent of the occupation of the other sublattice, as indicated by the $*$.

If there is a single bond between two different sites, for example, site $r$ and $s$, the effective bond energies are similar to the bond energy previously introduced, i.e.,

$$
\Delta^{\circ} G_{i: j: *: *}^{\varphi}=\varepsilon_{i j}^{r s}
$$

The formalism introduced here does not require a deep analysis of the number of different neighbors between each sites, as is traditionally the case for a bond energy model [32]. Such a refinement could however be used. The effective bond energies could then be expressed by

$$
\Delta^{\circ} G_{i: j: *: *}^{\varphi}=a_{s}^{\varphi} z_{s}^{r} \varepsilon_{i j}^{r s}
$$

where $z_{s}^{r}$ is the number of neighbors in site $r$ surrounding an atom on site $s$. The number of bonds between $s$ and $r$ sites is $a_{s}^{\varphi} z_{s}^{r}=a_{r}^{\varphi} z_{r}^{s}$. In some cases, like for fcc ordering, the assumption that $\varepsilon_{i j}^{r s}=\varepsilon_{j i}^{r s}$ could be added. It is, however, not a requirement of the model and would generally be incorrect. When considering the $\sigma$ 
phase, for instance, $\varepsilon_{A B}^{12}$ and $\varepsilon_{B A}^{12}$ would most of the time be very different because of different surroundings of the different sites. More generally, $\varepsilon_{i j}^{r s} \neq \varepsilon_{j i}^{r s}$ and $\varepsilon_{i j}^{r s} \neq \varepsilon_{i j}^{r t}$ for TCP phases. This is another significant difference compared to the traditional bond energy model.

The second set of summations in Eq. (11) considers contributions from triplets. The parameters of the type $\Delta^{\circ} G_{i: j: k: *}^{\varphi}$ are effective triplet energies. The third summation introduces effective tetrahedron energies. The parameters indicating more than two elements will generally be termed "many-body parameters." They are expected to be small and only used in the systems where enough experimental or theoretical information is available.

The term ${ }^{\mathrm{xs}} G^{\varphi}$ has the same expression as in Eq. (1). The expression of $\Delta G^{\varphi}\left(x_{i}^{\varphi}\right)$ is given by Eq. (5). Equations (1) and (4) with (11) are identical, provided that

$$
\begin{aligned}
G_{A: B: C: D}^{\varphi}= & a_{1}^{\varphi} G_{A}^{\varphi}+a_{2}^{\varphi} G_{B}^{\varphi}+a_{3}^{\varphi} G_{C}^{\varphi}+a_{4}^{\varphi} G_{D}^{\varphi} \\
& +\Delta^{\circ} G_{A: B: *: *}^{\varphi}+\Delta^{\circ} G_{A: *: C: *}^{\varphi}+\Delta^{\circ} G_{A: *: *: D}^{\varphi}+\Delta^{\circ} G_{*: B: C: *}^{\varphi}+\Delta^{\circ} G_{*: B: *: D}^{\varphi}+\Delta^{\circ} G_{*: *: C: D}^{\varphi} \\
& +\Delta^{\circ} G_{A: B: C: *}^{\varphi}+\Delta^{\circ} G_{A: B: *: D}^{\varphi}+\Delta^{\circ} G_{A: *: C: D}^{\varphi}+\Delta^{\circ} G_{*: B: C: D}^{\varphi}+\Delta^{\circ} G_{A: B: C: D}^{\varphi}
\end{aligned}
$$

The rest of this section will present the application of this formalism to two cases. The corresponding databases are available for downloading on the OC software web page [3], as this formalism is already available in this tool. The part corresponding to the phase treated with the new formalism for each example is given in Sec. 5.1.3 and Sec. 5.2.3.

The first example corresponds to the 4SL treatment of the fcc ordering, where the description presented with the new formalism is strictly identical to the original one [28]. The second example is presented in more detail to show the potential of the new formalism for the $\sigma$ phase case where only the effective bond energies are considered.

\subsection{Al-Cr-Ni fec ordering}

When considering the fcc ordering $\left(A 1, L 1_{2}\right.$, and $\left.L 1_{0}\right)$ with 4 SLs in the EBEF, Eq. (14) becomes:

$$
\begin{aligned}
G_{A: B: C: D}^{4 S L}= & \frac{1}{4} G_{A}^{A 1}+\frac{1}{4} G_{B}^{A 1}+\frac{1}{4} G_{C}^{A 1}+\frac{1}{4} G_{D}^{A 1} \\
& +\Delta^{\circ} G_{A: B: *: *}^{4 S L}+\Delta^{\circ} G_{A:: C: *}^{4 S L}+\Delta^{\circ} G_{A: *: *: D}^{4 S L}+\Delta^{\circ} G_{*: B: C: *}^{4 S L}+\Delta^{\circ} G_{*: B: *: D}^{4 S L}+\Delta^{\circ} G_{*: *: C: D}^{4 S L} \\
& +\Delta^{\circ} G_{A: B: C: *}^{4 S L}+\Delta^{\circ} G_{A: B: *: D}^{4 S L}+\Delta^{\circ} G_{A: *: C: D}^{S L L}+\Delta^{\circ} G_{*: B: C: D}^{4 S L}+\Delta^{\circ} G_{A: B: C: D}^{S L L}
\end{aligned}
$$

Using the bond energy notation, it yields:

$$
\begin{aligned}
G_{A: B: C: D}^{4 S L}= & \frac{1}{4} G_{A}^{A 1}+\frac{1}{4} G_{B}^{A 1}+\frac{1}{4} G_{C}^{A 1}+\frac{1}{4} G_{D}^{A 1} \\
& +\varepsilon_{A B}^{12}+\varepsilon_{A C}^{13}+\varepsilon_{A D}^{14}+\varepsilon_{B C}^{23}+\varepsilon_{B D}^{24}+\varepsilon_{C D}^{34} \\
& +\varepsilon_{A B C}^{123}+\varepsilon_{A B D}^{124}+\varepsilon_{A C D}^{134}+\varepsilon_{B C D}^{234}+\varepsilon_{A B C D}^{1234}
\end{aligned}
$$

Due to the symmetry of the crystallographic structure, it can be rewritten:

$$
\begin{aligned}
G_{A: B: C: D}^{4 S L}= & \frac{1}{4} G_{A}^{A 1}+\frac{1}{4} G_{B}^{A 1}+\frac{1}{4} G_{C}^{A 1}+\frac{1}{4} G_{D}^{A 1} \\
& +\varepsilon_{A B}+\varepsilon_{A C}+\varepsilon_{A D}+\varepsilon_{B C}+\varepsilon_{B D}+\varepsilon_{C D} \\
& +\varepsilon_{A B C}+\varepsilon_{A B D}+\varepsilon_{A C D}+\varepsilon_{B C D}+\varepsilon_{A B C D}
\end{aligned}
$$

A database using the EBEF for the $\gamma^{\prime}$ phase of the Al-Cr-Ni system has been created. The part corresponding to the ordering of this phase is given in Sec. 5.1.3. It should be noted that with some Calphad 
software, the symmetry of this phase can be taken into account by using the F option, i.e., adding ":F" after the name of the phase. This allows the equivalent parameters to be entered into the thermodynamic database file only once. There are only five in this case. In addition to the effective bond energies, $\Delta^{\circ} G_{A: B: *: *}^{4 S L}$, defined in each binary system, an excess interaction parameter is used for the Al-Ni system, $L_{A l, N i: *: *: *}^{4 S L}$ and a correction is used for the Gibbs energy of the ternary compound $\mathrm{AlCrNi}_{2}, \Delta G_{A l: C r: N i: N i}^{4 S L}$. This database has been tested with the OC software [3] and yields results that are identical to the original description by Dupin et al. [28], which uses a 2SL CEF with 29 parameters. The ordering part of this description is listed in Sec. 5.1.1. The parameters are expressed as functions corresponding to the ordering parameters of an equivalent symmetrical 4SL CEF. The description with the 4SL CEF where the symmetry of the structure is considered (F option) allows definition of only 13 parameters, as shown in Sec. 5.1.2. The increase in the number of parameters corresponding to the Gibbs energy of the end members is more than compensated by the decrease of the number of interaction parameters.

The simplicity of the EBEF description is striking. Even if it is mathematically equivalent to the 2SL $\mathrm{CEF}$ that is currently used in a number of multicomponent databases, its use has great potential to significantly simplify multicomponent database management.

\subsection{Mo-Ni-Re $\sigma$ phase}

In order to assess the ability of the new formalism to extrapolate into higher-order systems, we will revisit the Mo-Ni-Re ternary example already used in Section 2.2 to show the weakness of the CEF extrapolation for complex phases.

Effective bond energies for the $\sigma$ phase were first derived in the three constitutive binary systems using only the binary DFT results [22]. For a given binary system, the Gibbs energies of formation of the 30 stoichiometric end member compounds, $\sigma_{i}$, are expressed as functions of 20 possible effective bond energies, depending of their configuration, as follows:

$$
\begin{aligned}
G_{A: B: C: D: E}^{\sigma_{i}}= & 4 G_{A}^{\sigma}+2 G_{B}^{\sigma}+8 G_{C}^{\sigma}+8 G_{D}^{\sigma}+8 G_{E}^{\sigma}+\Delta^{\circ} G_{A: B: *: * * *}^{\sigma} \\
& +\Delta^{\circ} G_{A: *: C: *: *}^{\sigma}+\Delta^{\circ} G_{A: *: *: D: *}^{\sigma}+\Delta^{\circ} G_{A: *: *: * E}^{\sigma} \\
& +\Delta^{\circ} G_{*: B: C: *: *}^{\sigma}+\Delta^{\circ} G_{*: B: *: D: *}^{\sigma}+\Delta^{\circ} G_{*: B: *: * E}^{\sigma} \\
& +\Delta^{\circ} G_{*: *: C: D: *}^{\sigma}+\Delta^{\circ} G_{*: *: C: *: E}^{\sigma}+\Delta^{\circ} G_{*: *:: D: E}^{\sigma}
\end{aligned}
$$

The 20 effective bond energies then were optimized with the PARROT module of the Thermo-Calc software [33] to fit the DFT results for the 30 binary configurations (compounds), and the results are reported in Table 2 for each binary system. The values for the 30 stoichiometric compounds in each binary system are compared in Fig. 4. The abscissa corresponds to the DFT results, and the ordinate corresponds to the values obtained with the assessed effective bond energies listed in Table 2. Excellent agreement was obtained for all Mo-Re compounds. The agreement in the Ni-Re and Mo-Ni systems was not as good. For fitting the EBEF parameters, higher weights were given to the compounds with a significant contribution to the stable configuration, i.e., negative energies. The DFT values for the different compounds of Mo-Ni varied significantly more than those for $\mathrm{Ni}-\mathrm{Re}$, which varied slightly more than those for Mo-Re. This has been attributed to the difference in atomic size, with Ni being the smallest atom and Re only slightly smaller than Mo [22]. In addition, the DFT calculations were carried out without spin-polarization, and it is possible that the difference in the energy between the ferromagnetic and nonmagnetic state for Ni-rich end members also affected the fit of the EBEF parameters. It is also possible that a large variation in compound energies requires consideration of more clusters, and closer agreement could be obtained if larger clusters, such as triplets, were employed. However, the need for larger clusters should be evaluated in the course of a Calphad assessment of the description of a binary or ternary system. 


\section{Journal of Research of National Institute of Standards and Technology}

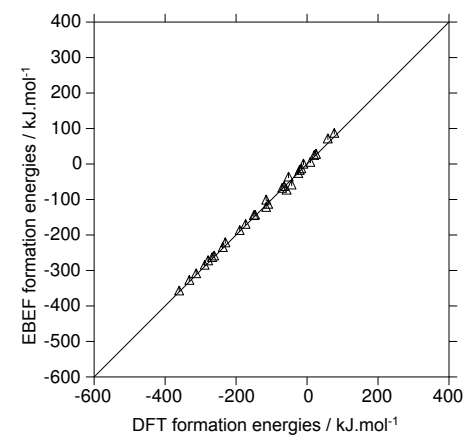

Mo-Re

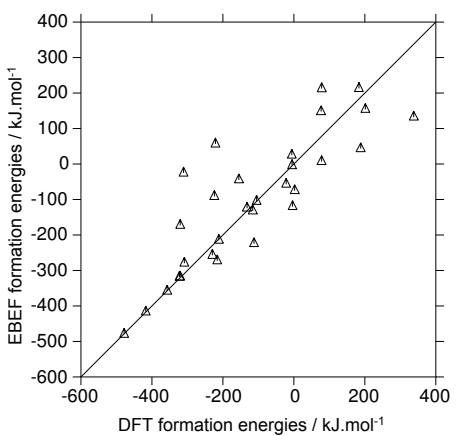

Mo-Ni

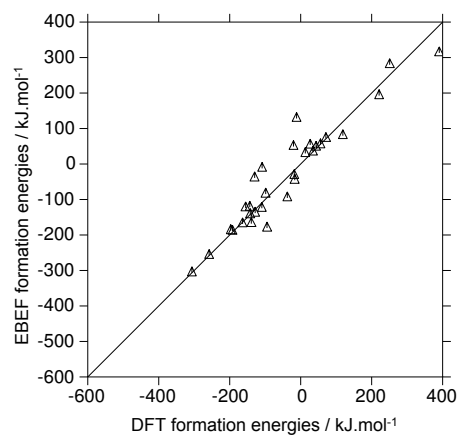

$\mathrm{Ni}-\mathrm{Re}$

Fig. 4. Formation energies of the binary stoichiometric $\sigma$ configurations from the pure elements in the $\sigma$ structure. For each compound, the $x$ coordinate corresponds to the DFT results [22], and the $y$ coordinate corresponds to the values calculated by Eq. (18) with the effective bond energies listed in Table 2. The diagonal line indicates the perfect agreement between the two sets of values.

Table 2. Effective bond energies, in $\mathrm{J} / \mathrm{mol}$ (mole of formula unit), derived in the present work. For each binary system, the 20 effective bond energies were optimized to fit the DFT results for the 30 binary configurations (compounds).

\begin{tabular}{|c|c|c|c|}
\hline A-B & Mo-Re & Mo-Ni & $\mathrm{Ni}-\mathrm{Re}$ \\
\hline$\Delta^{\circ} G_{A: B: *: *: *}^{\sigma}$ & +680 & -112309 & -103625 \\
\hline$\Delta^{\circ} G_{A: *: B: *: *}^{\sigma}$ & +16094 & +29525 & +20648 \\
\hline$\Delta^{\circ} G_{A: *: *: B: *}^{\sigma}$ & 0 & -965 & -88284 \\
\hline$\Delta^{\circ} G_{A: *: *: *: B}^{\sigma}$ & +4361 & -40910 & +32709 \\
\hline$\Delta^{\circ} G_{*: A: B: *: *}^{\sigma}$ & -14434 & -109029 & -46120 \\
\hline$\Delta^{\circ} G_{*: A: *: B: *}^{\sigma}$ & -81839 & -134018 & +91951 \\
\hline$\Delta^{\circ} G_{*: A: *: * B}^{\sigma}$ & -28743 & -2219 & +36182 \\
\hline$\Delta^{\circ} G_{*: *: A: B: *}^{\sigma}$ & -86837 & -167595 & +70531 \\
\hline$\Delta^{\circ} G_{*: *: A: *: B}^{\sigma}$ & -73358 & +66189 & +136624 \\
\hline$\Delta^{\circ} G_{*: *: *: A: B}^{\sigma}$ & +21024 & -16596 & -77381 \\
\hline$\Delta^{\circ} G_{B: A: *: *: *}^{\sigma}$ & -22076 & -73811 & -2123 \\
\hline$\Delta^{\circ} G_{B: *: A: *: *}^{\sigma}$ & -19297 & -7043 & -19973 \\
\hline$\Delta^{\circ} G_{B: *: *: A: *}^{\sigma}$ & +5847 & -41875 & -1351 \\
\hline$\Delta^{\circ} G_{B: *: *: * A}^{\sigma}$ & -30827 & +17271 & -61558 \\
\hline$\Delta^{\circ} G_{*: B: A: *: *}^{\sigma}$ & +6571 & +82688 & +5210 \\
\hline$\Delta^{\circ} G_{*: B: *: A: *}^{\sigma}$ & +42569 & +63970 & -89153 \\
\hline$\Delta^{\circ} G_{*: B: *: *: A}^{\sigma}$ & -25482 & +97740 & -2026 \\
\hline$\Delta^{\circ} G_{*: *: B: A: *}^{\sigma}$ & -1467 & +19008 & -89828 \\
\hline$\Delta^{\circ} G_{*: *: B: *: A}^{\sigma}$ & -62108 & +55865 & +75934 \\
\hline$\Delta^{\circ} G_{*: *: *: B: A}^{\sigma}$ & -119768 & -114625 & -24700 \\
\hline
\end{tabular}




\section{Journal of Research of National Institute of Standards and Technology}
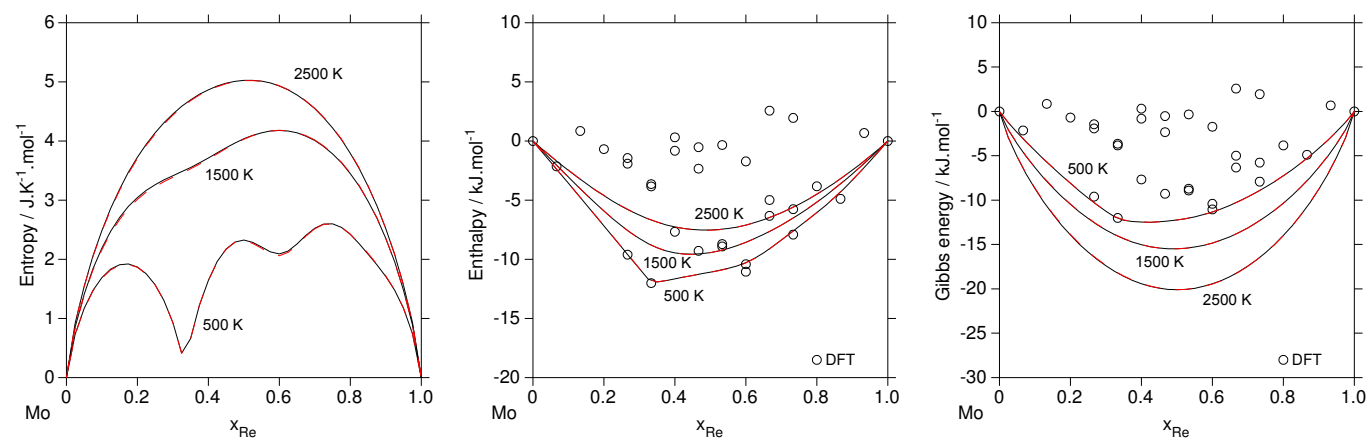

Fig. 5. Comparison of the $\sigma$ thermodynamic properties in the Mo-Re binary system calculated for $500 \mathrm{~K}, 1500 \mathrm{~K}$, and $2500 \mathrm{~K}$ using the CEF with 30 stoichiometric compounds from DFT results [22] (shown in red dashed lines) and the EBEF using 20 effective bond energies (Table 2, second column, shown in black solid lines).
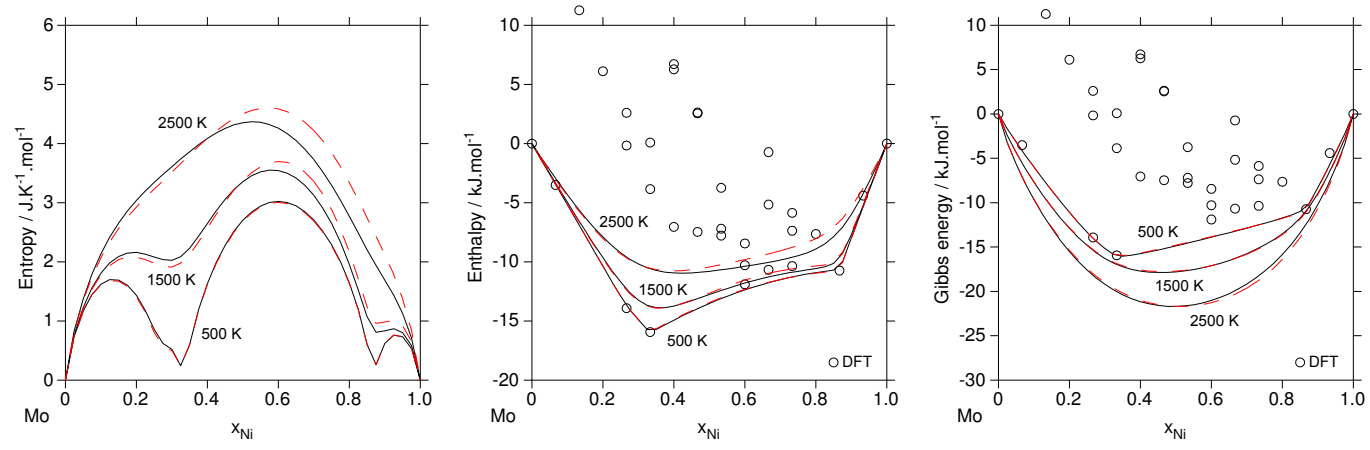

Fig. 6. Comparison of the $\sigma$ thermodynamic properties in the Mo-Ni binary system calculated for $500 \mathrm{~K}, 1500 \mathrm{~K}$, and $2500 \mathrm{~K}$ using the CEF with 30 stoichiometric compounds from DFT results [22] (shown in red dashed lines) and the EBEF using 20 effective bond energies (Table 2, third column, shown in black solid lines).
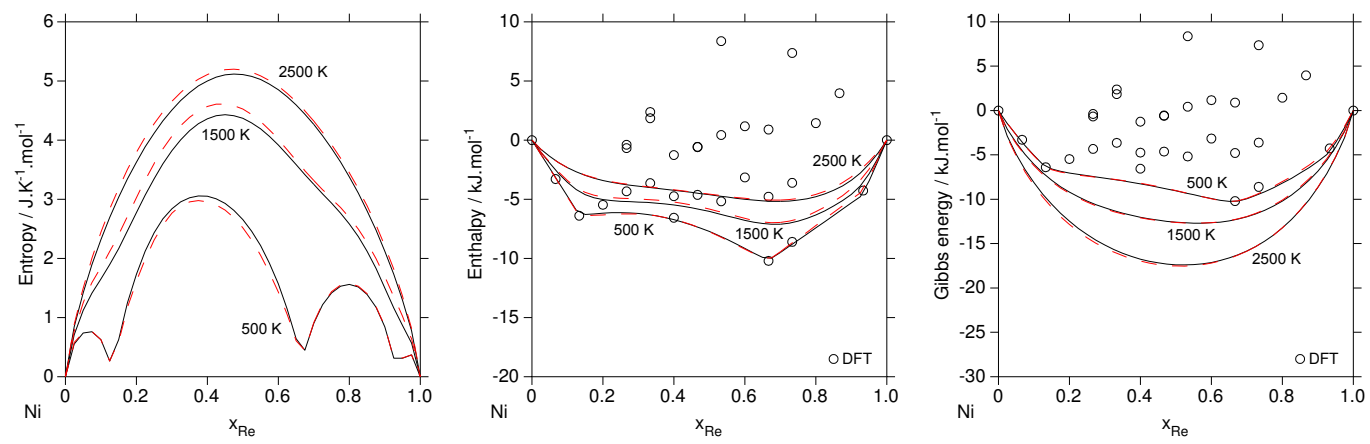

Fig. 7. Comparison of the $\sigma$ thermodynamic properties in the Ni-Re binary system calculated for $500 \mathrm{~K}, 1500 \mathrm{~K}$, and $2500 \mathrm{~K}$ using the CEF with 30 stoichiometric compounds from DFT results [22] (shown in red dashed lines) and the EBEF using 20 effective bond energies (Table 2, fourth column, shown in black solid lines). 


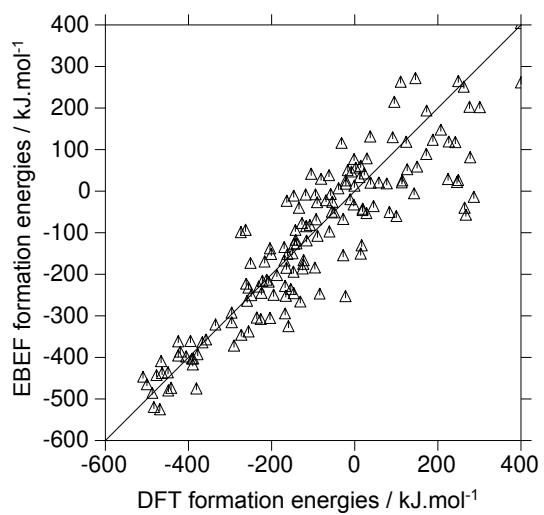

Fig. 8. Formation energies of the ternary stoichiometric $\sigma$ configurations from the pure elements in the $\sigma$ structure. For each compound, the $x$ coordinate corresponds to the DFT results [22], and the $y$ coordinate corresponds to the values calculated by Eq. (18) with the effective bond energies listed in Table 2. The diagonal line indicates the perfect agreement between the two sets of values.

The good agreement obtained for all the stoichiometric compounds in the case of Mo-Re allows the thermodynamic properties calculated at different temperatures with the two models to be very close over the entire compositional range (Fig. 5). The calculated binary phase diagram, not shown here, is almost identical to the one using the DFT values for the binary compounds of this system [17]. In the cases of Mo-Ni and Ni-Re (Figs. 6 and 7), the rather poor fit of some of the configurations results in notable differences in the thermodynamic properties at high temperatures.

The values for the 150 ternary compounds calculated with the EBEF description are compared to the DFT results in Fig. 8. The agreement is about the same as for the binary Mo-Ni system, which indicates that the quality of the ternary prediction using EBEF depends strongly on the quality of the fit of the binary compounds.

The ternary $\sigma$ EBEF description using the effective bond energies derived in the three binary systems is given in Sec. 5.2.3. It can currently only be used with the OC software [3]. The isothermal sections calculated with OC are shown in Fig. 9. An equivalent description using the CEF implementation is given in Sec. 5.2.4. It can be used in other software supporting the thermodynamic database format where the EBEF is not yet implemented; the results are shown in Fig. 10. The number of parameters in these descriptions is respectively 60 and 240. The only differences between Fig. 9 and Fig. 10 are the layout of the plot and the spacing of the tie lines.

The two sets of phase diagrams in Fig. 2 and Fig. 10 are very similar, with only minor differences being noticeable. This result is very satisfactory, considering that only binary information has been used in the EBEF description. At $500 \mathrm{~K}$, the EBEF predicts the stabilization of the $\sigma$ phase and a miscibility gap inside the $\sigma$ phase, similar to the description using all of the compound values from DFT. At $1500 \mathrm{~K}$ and $2500 \mathrm{~K}$, the limit of the extension of the $\sigma$ phase calculated with the EBEF using only binary effective bond energies (Fig. 10) is very close to the description with the CEF using the description of 243 end members (Fig. 2).

In contrast, the difference between the calculated phase diagrams using only binary parameters in Fig. 1 (CEF) and Fig. 10 (EBEF) is tremendous. In the diagrams calculated with the CEF, the $\sigma$ phase extends barely into the ternary system at $1500 \mathrm{~K}$ and $2500 \mathrm{~K}$ and does not occur at all at $500 \mathrm{~K}$, as it does when using the EBEF. This illustrates very clearly the improvement introduced by the EBEF when extrapolating 


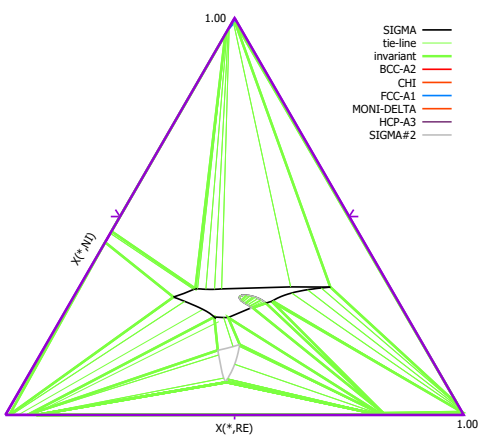

$\mathrm{T}=500 \mathrm{~K}$

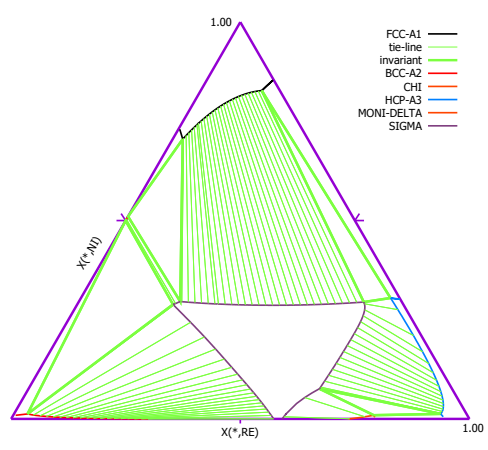

$\mathrm{T}=1500 \mathrm{~K}$

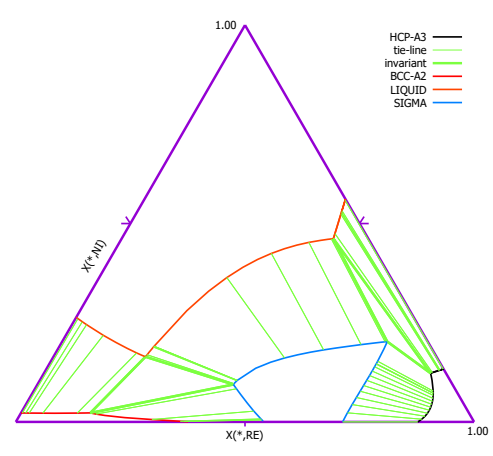

$\mathrm{T}=2500 \mathrm{~K}$

Fig. 9. Isothermal sections in the Mo-Ni-Re system calculated for $500 \mathrm{~K}, 1500 \mathrm{~K}$, and $2500 \mathrm{~K}$ with the EBEF using 20 effective bond energies from each binary system using OpenCalphad [3], with the description in Sec. 5.2.3.

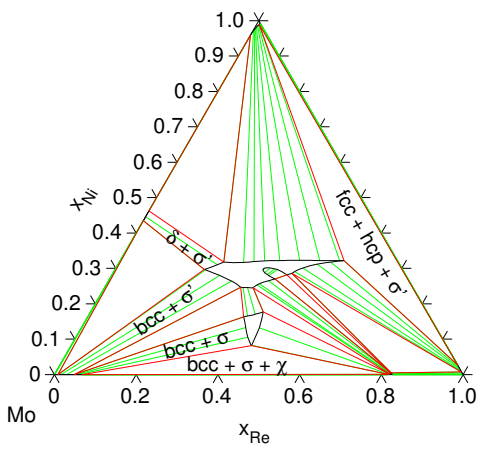

$\mathrm{T}=500 \mathrm{~K}$

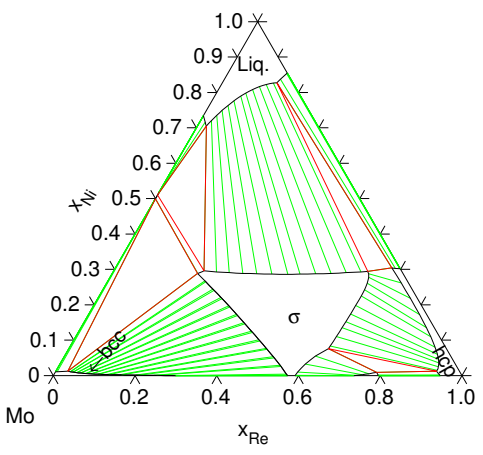

$\mathrm{T}=1500 \mathrm{~K}$

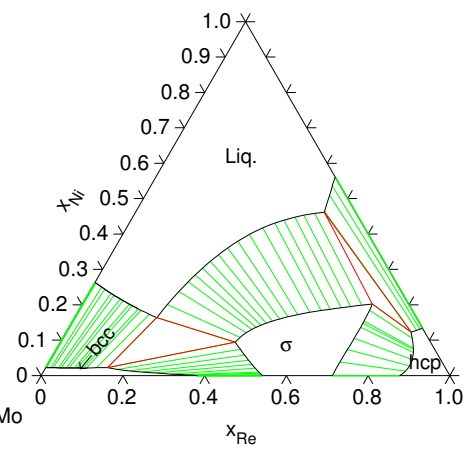

$\mathrm{T}=2500 \mathrm{~K}$

Fig. 10. Isothermal sections in the Mo-Ni-Re system calculated for $500 \mathrm{~K}, 1500 \mathrm{~K}$, and $2500 \mathrm{~K}$ with the CEF equivalent to the EBEF using 20 effective bond energies from each binary system using Thermo-Calc [33], with the description in Sec. 5.2.4. 
from the binary systems into higher-order systems. These first results show that this model can satisfactorily describe binary interactions while keeping the complexity of the crystallographic structure and thus allowing description of the behavior of complex phases in multicomponent systems.

It needs to be emphasized that the present calculations do not represent a thermodynamic assessment of the Mo-Ni-Re system, because the disordered solution phases are described without any ternary interaction parameters, and no ternary experimental phase diagram data were taken into consideration.

\section{Conclusion}

The new formalism presented here is based on the decomposition of the Gibbs energies of the end member compounds of a phase into effective bond energies of the pairs involved in its configuration. The extra contribution from many-body interactions is also considered. Although this formalism is mathematically identical to the widely used CEF, it has been shown here that it brings several important improvements. It allows for models to more closely correspond to the real crystallography of the phases, even for complex structures, while giving better extrapolations to higher-order systems. Finally, it opens the possibility for significant simplification of multicomponent thermodynamic databases, greatly reducing the need for the large number of DFT calculations that are required for the development of accurate descriptions, resulting in better extrapolations. The full potential or limitations of this new formalism still need to be further explored by applying it to phases with a different crystal structure and exploring how the effective binary bonds are possibly influenced in systems with very different elements, in particular, those with different magnetic properties.

\section{Appendix}

The following sections present extracts of the databases corresponding to the application of the new formalism to the Al-Cr-Ni and Mo-Ni-Re systems.

\subsection{Al-Cr-Ni fec ordering}

\subsubsection{CEF-2SL}

This description corresponds to the original publication [28]. It is identical to those in Sec. 5.1.2 and Sec. 5.1.3 for the stable equilibria but not for the metastable states involving the $L 1_{0}$ ordering because it cannot describe the $L 1_{0}$ ordering. There are no $G$ parameters for the pure elements, since these parameters are referred to the pure elements in the disordered contribution in this description. That contribution is not given here, but it appears in the TDB file (AlCrNi_CEF_2SL.TDB) provided as supplemental material.

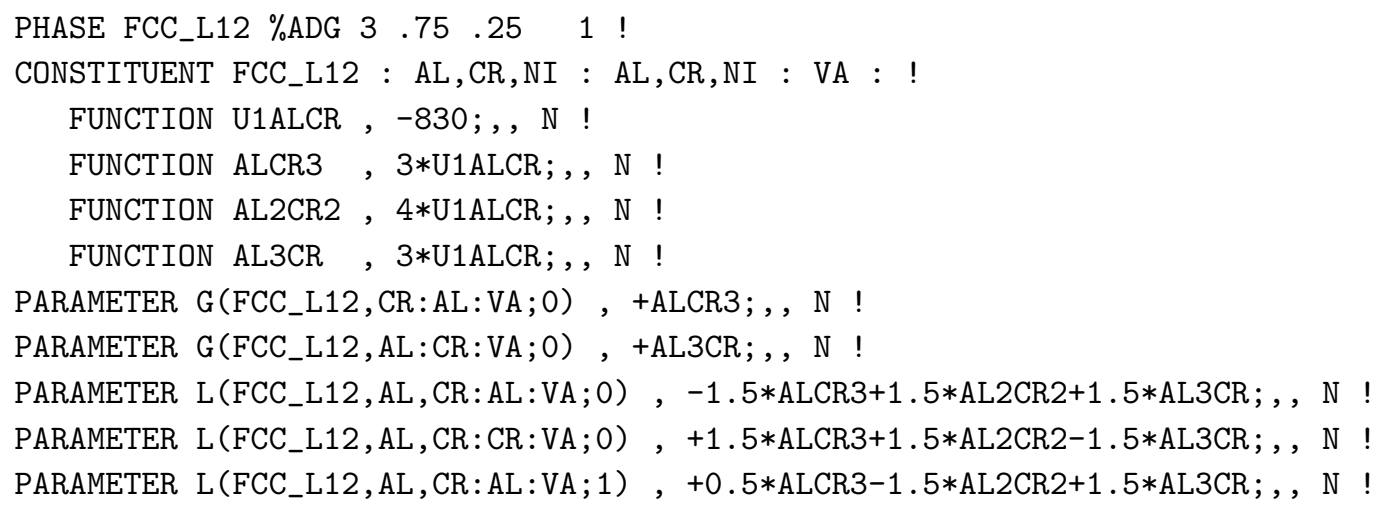




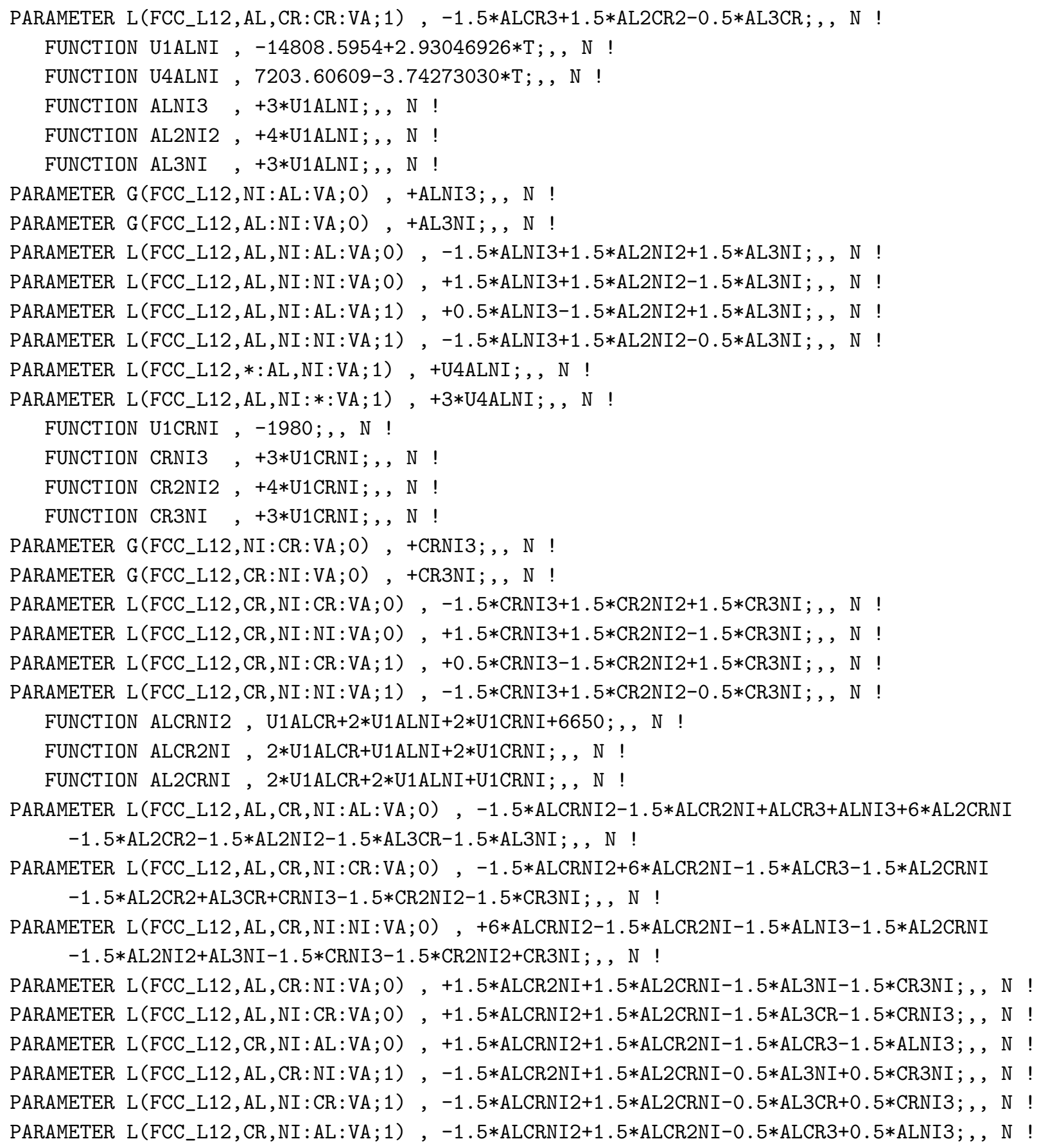

\subsubsection{CEF-4SL}

See files AlCrNi_CEF_4SL.TDB and AlCrNi_CEF_4SLF (using F option) in the supplemental materials.

PHASE FCC_L12:F \%ADG $5 \quad .25 \quad .25 \quad .25 \quad .25 \quad 1 \quad$ !

CONSTITUENT FCC_L12:F : AL,CR,NI : AL,CR,NI: AL,CR,NI: AL,CR,NI : VA : !

FUNCTION U1ALCR , $-830 ;,$, N ! 


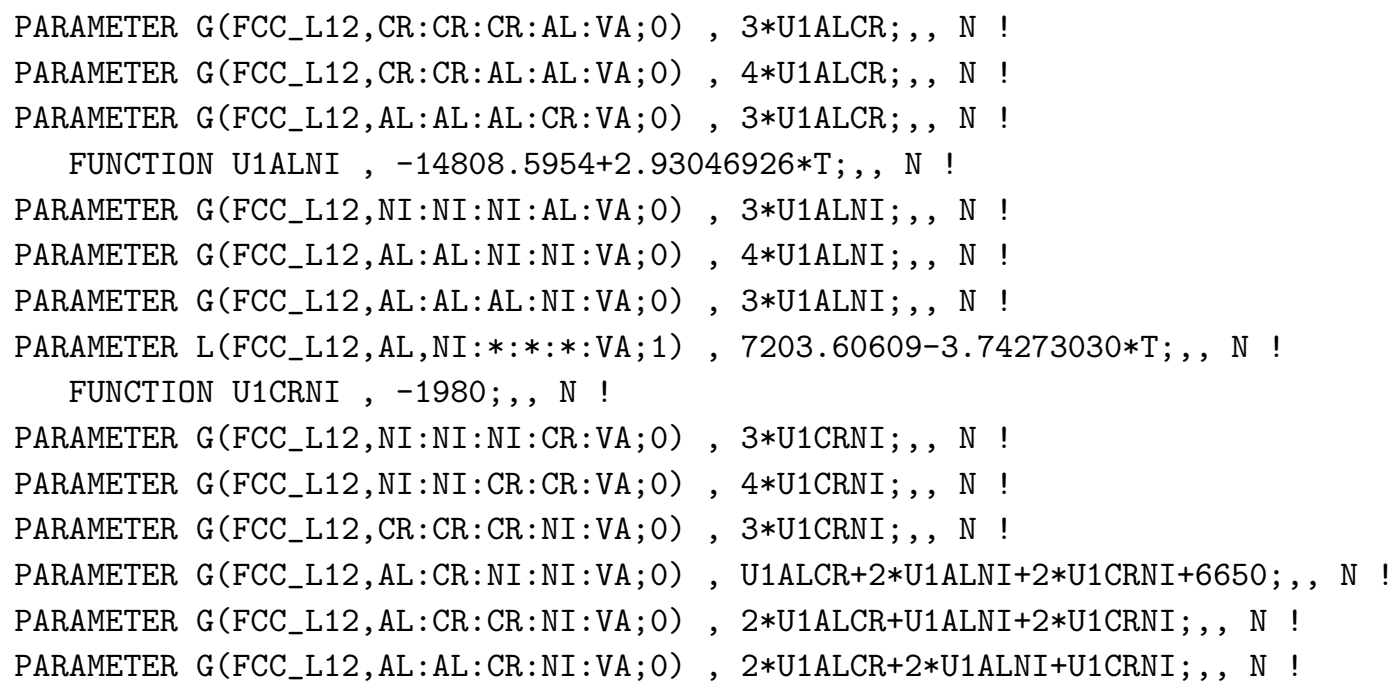

\subsubsection{EBEF}

This description is strictly identical to the one in Sec. 5.1.2 (AlCrNi_BEF.TDB in the supplemental materials).

PHASE FCC_L12:F \%ADG $5 \quad .25 \quad .25 \quad .25 \quad .25 \quad 1 \quad$ !

CONSTITUENT FCC_L12:F : AL, CR,NI : AL, CR,NI: AL,CR,NI: AL, CR,NI : VA : !

PARAMETER G(FCC_L12,AL:CR:*:*:VA;0) , -830;, , N !

PARAMETER G(FCC_L12,AL:NI:*:*:VA;0) , $-14808.5954+2.93046926 * \mathrm{~T} ;$, , N !

PARAMETER L(FCC_L12,AL,NI:*:*:*:VA;1) , 7203.60609-3.74273030*T; , , N !

PARAMETER G(FCC_L12,CR:NI:*:*:VA;0), $-1980 ;,$, N !

PARAMETER G(FCC_L12,AL:CR:NI:NI:VA;0), 6650;, ,N !

\subsection{Mo-Ni-Re $\sigma$ phase}

\subsubsection{DFT-CEF with only binary compounds}

The 90 binary parameters are set to the DFT results in this description. They correspond to the energies of formation from the pure elements with $\sigma$ structure. The lattice stabilities of the three pure elements in the $\sigma$ phase with respect to the SER states are given as parameters of the configuration-independent contribution that is added to this configuration-dependent contribution. The parameters of the configuration-independent contribution are identical to those of the other descriptions in Secs. 5.2.2, 5.2.4, and 5.2.3. They are not presented here but appear in the TDB file (MoNiRe_CEF_b.TDB), given as supplemental material together with the description of all the other stable phases in the system.

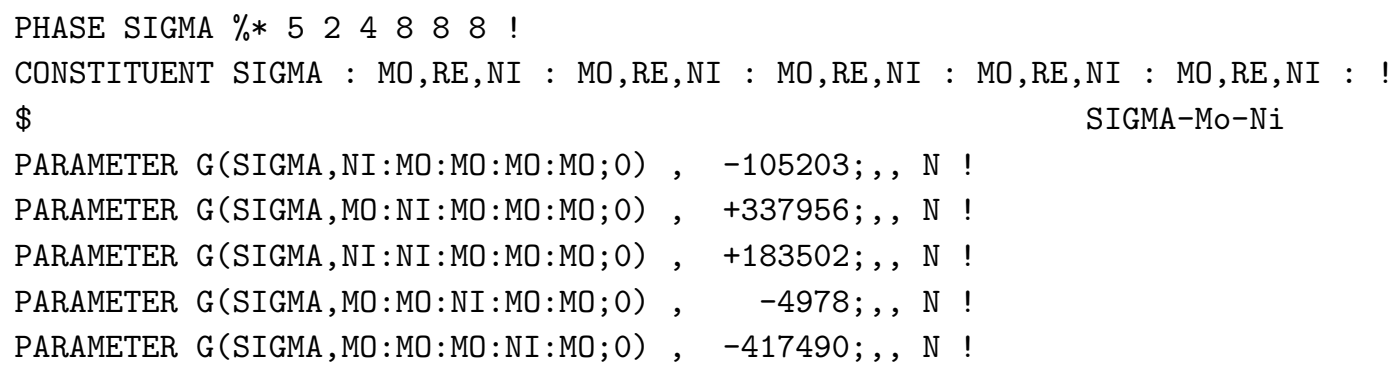




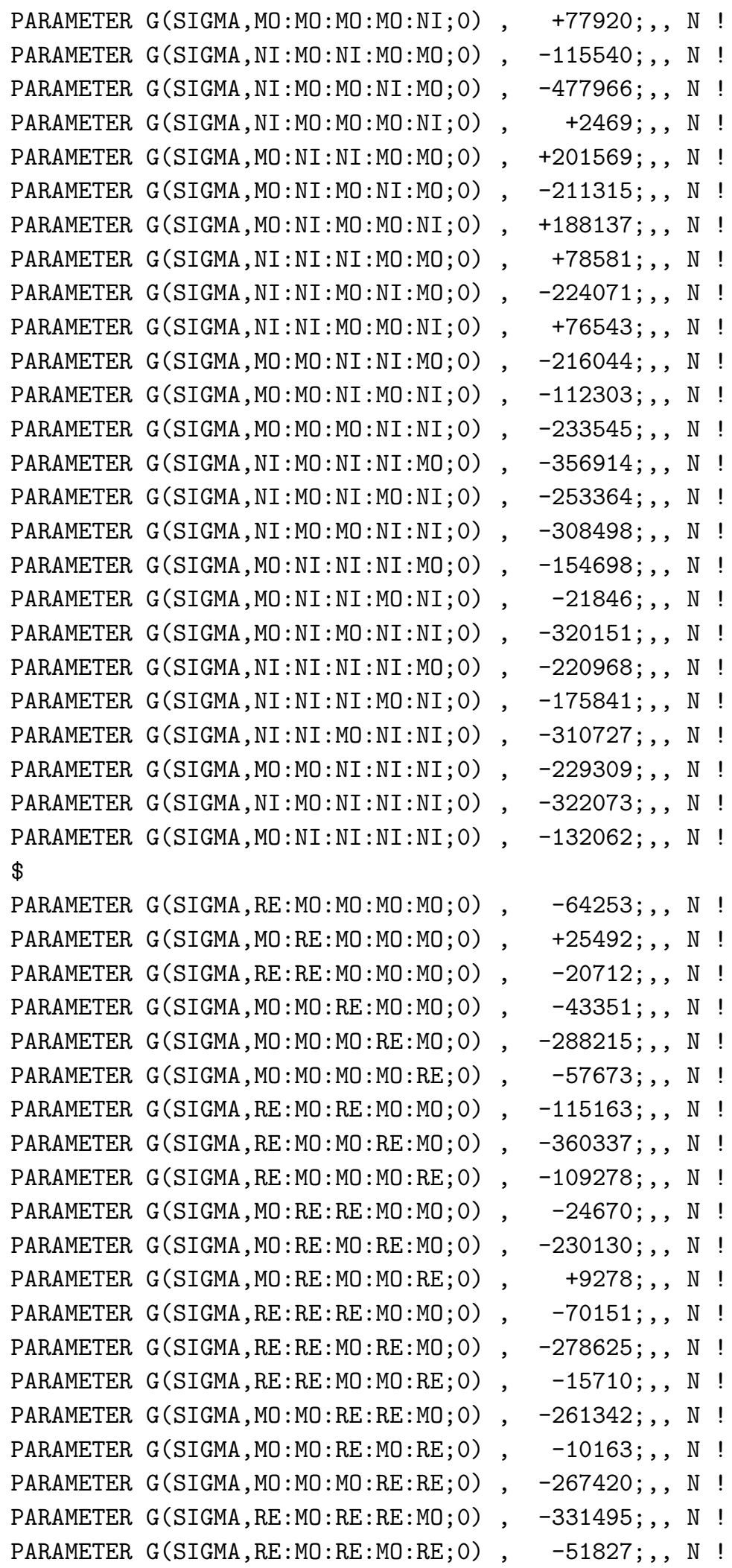




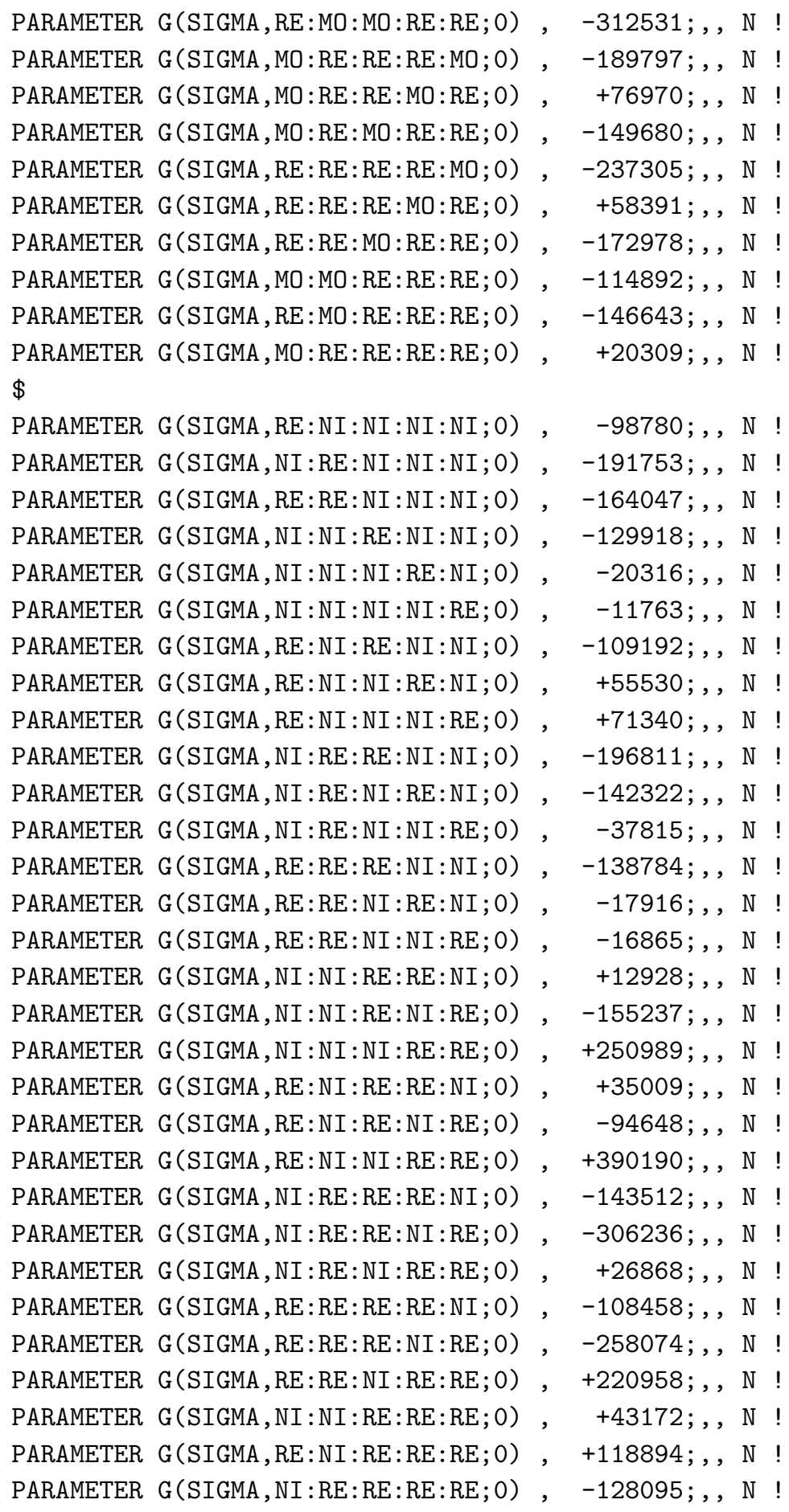

\subsubsection{DFT-CEF with binary and ternary compounds}

In this description, the 90 binary and 150 ternary parameters are set to the DFT results. Since the phase description and binary parameters are identical to those in Sec. 5.2.1, only the ternary parameters are listed here. The complete description is given in the TDB file (MoNiRe_CEF_t.TDB) in the supplemental 
materials.

$\$$

PARAMETER G(SIGMA,NI:RE:MO:MO:MO;0), -89607;, , N ! PARAMETER G(SIGMA,NI:MO:MO:RE:MO;0), -449430;, , N ! PARAMETER G(SIGMA,NI:RE:RE:MO:MO;0), -149941;, N ! PARAMETER G(SIGMA,NI:RE:MO:RE:MO;0), -391643;, , N ! PARAMETER G(SIGMA,NI:MO:MO:MO:RE;0), -168881;, N ! PARAMETER G(SIGMA,NI:RE:MO:MO:RE;0), -115752;, N ! PARAMETER G(SIGMA,NI:MO:RE:MO:RE;0), -107779;, , N ! PARAMETER G(SIGMA,NI:RE:RE:MO:RE;0), $\quad-53950 ;, \mathrm{N}$ ! PARAMETER G(SIGMA,NI:MO:RE:RE:MO;0), -405677;,, N ! PARAMETER G(SIGMA,NI:MO:MO:RE:RE;0), -388780;,, N ! PARAMETER G(SIGMA,NI:RE:RE:RE:MO;0), -357540;, N ! PARAMETER G(SIGMA,NI:RE:MO:RE:RE;0), -295879;, N ! PARAMETER G(SIGMA,NI:MO:RE:RE:RE;0), -230704;, N ! PARAMETER G(SIGMA,NI:MO:RE:MO:MO;0), -162107;, , N ! PARAMETER G(SIGMA,MO:NI:RE:MO:RE;0), +286359;, N ! PARAMETER G(SIGMA,RE:NI:RE:MO:RE;0), +277521;, N ! PARAMETER G(SIGMA,RE:NI:RE:MO:MO;0), +224127;, N ! PARAMETER G(SIGMA,MO:NI:RE:MO:MO;0), +264370;,, N ! PARAMETER G(SIGMA,MO:NI:RE:RE:MO;0), +16458;,, N ! PARAMETER G(SIGMA,RE:NI:RE:RE:MO;0), -28016;,, N ! PARAMETER G(SIGMA,RE:NI:MO:MO:MO;0), +300656;, , N ! PARAMETER G(SIGMA,MO:NI:MO:MO:RE;0) , PARAMETER G(SIGMA,MO:NI:MO:RE:RE;0) , PARAMETER G(SIGMA,RE:NI:MO:RE:RE;0) , PARAMETER G(SIGMA,RE:NI:MO:MO:RE;0), PARAMETER G(SIGMA,MO:NI:MO:RE:MO;0), PARAMETER G(SIGMA,MO:NI:RE:RE:RE;0), PARAMETER G(SIGMA,RE:NI:MO:RE:MO;0), PARAMETER G(SIGMA,NI:NI:MO:RE:RE;0) , PARAMETER G(SIGMA,NI:NI:RE:RE:MO;0), PARAMETER G(SIGMA,NI:NI:RE:MO:MO;0), PARAMETER G(SIGMA,NI:NI:MO:MO:RE;0), PARAMETER G(SIGMA,NI:NI:MO:RE:MO;0), PARAMETER G(SIGMA,NI:NI:RE:MO:RE;0), PARAMETER G(SIGMA,RE:MO:NI:MO:RE;0), PARAMETER G(SIGMA,MO:RE:NI:MO:RE;0), PARAMETER G(SIGMA,RE:RE:NI:MO:RE;0), PARAMETER G(SIGMA,MO:RE:NI:RE:MO;0), PARAMETER G(SIGMA,RE:RE:NI:RE:MO;0), PARAMETER G(SIGMA,MO:RE:NI:MO:MO;0), PARAMETER G(SIGMA,MO:MO:NI:RE:MO;0), PARAMETER G(SIGMA,RE:RE:NI:MO:MO;0), PARAMETER G(SIGMA,RE:MO:NI:MO:MO;0), PARAMETER G(SIGMA,MO:MO:NI:RE:RE;0),
SIGMA Mo-Ni-Re

$+248780 ;,$, N !

$+28115 ;,$, N !

$+11444 ;,$, N !

$+225931 ;$, , N !

+18916; , , N !

+142599; , , N !

-21373 ; , , N !

$-94661 ;,$ N !

$-116818 ;,$, N !

+126297; , , N !

+123866; , , N !

-118039 ; , , N !

+172022; , , N !

$-39274 ;,$, N !

+110584; , , N !

+95132;,, N !

+113135; , , N !

+100205; , , N !

+37033; , N !

+14320 ; , , N !

+2226; , , N !

$-60866 ;,, \mathrm{N}$ !

+114587; , , N ! 


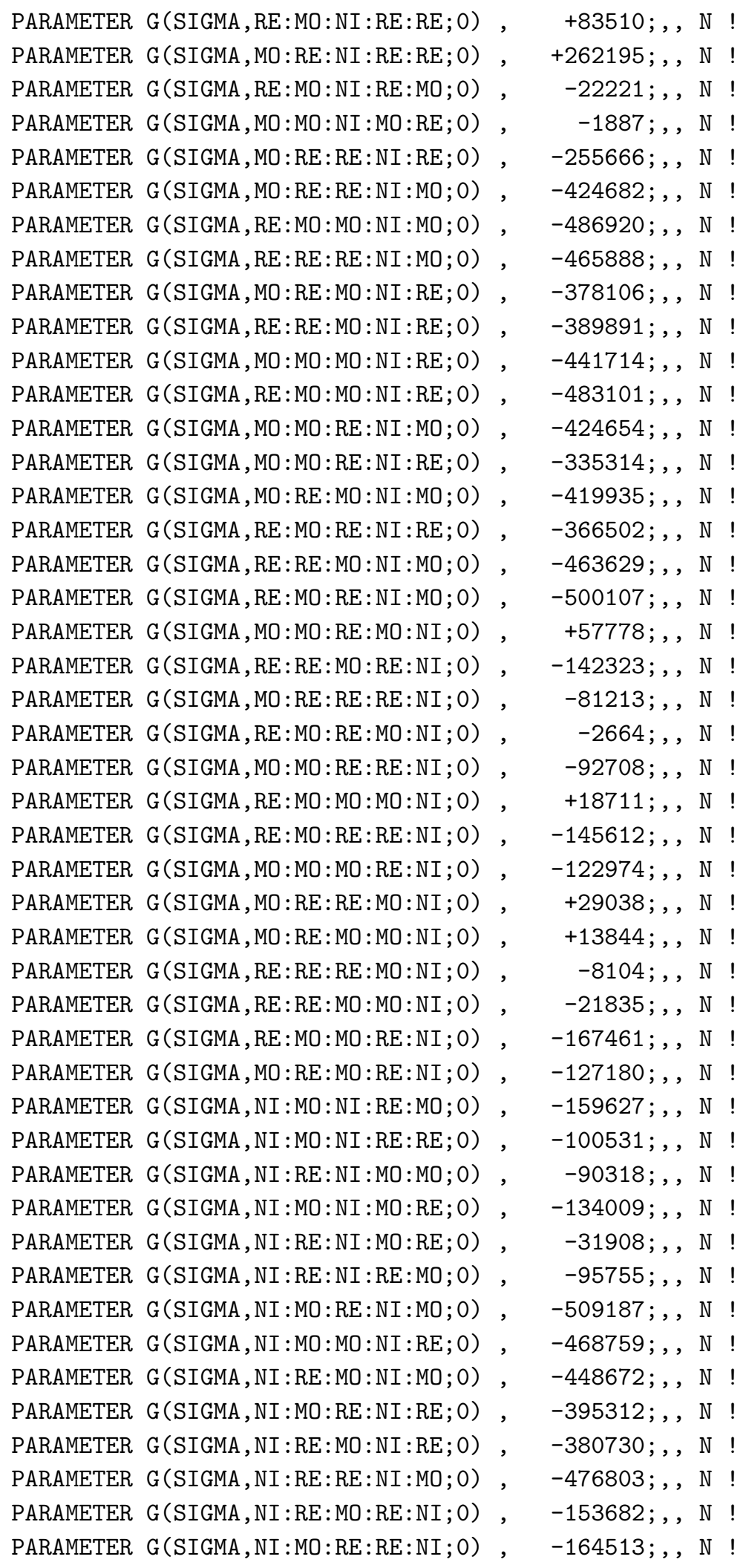




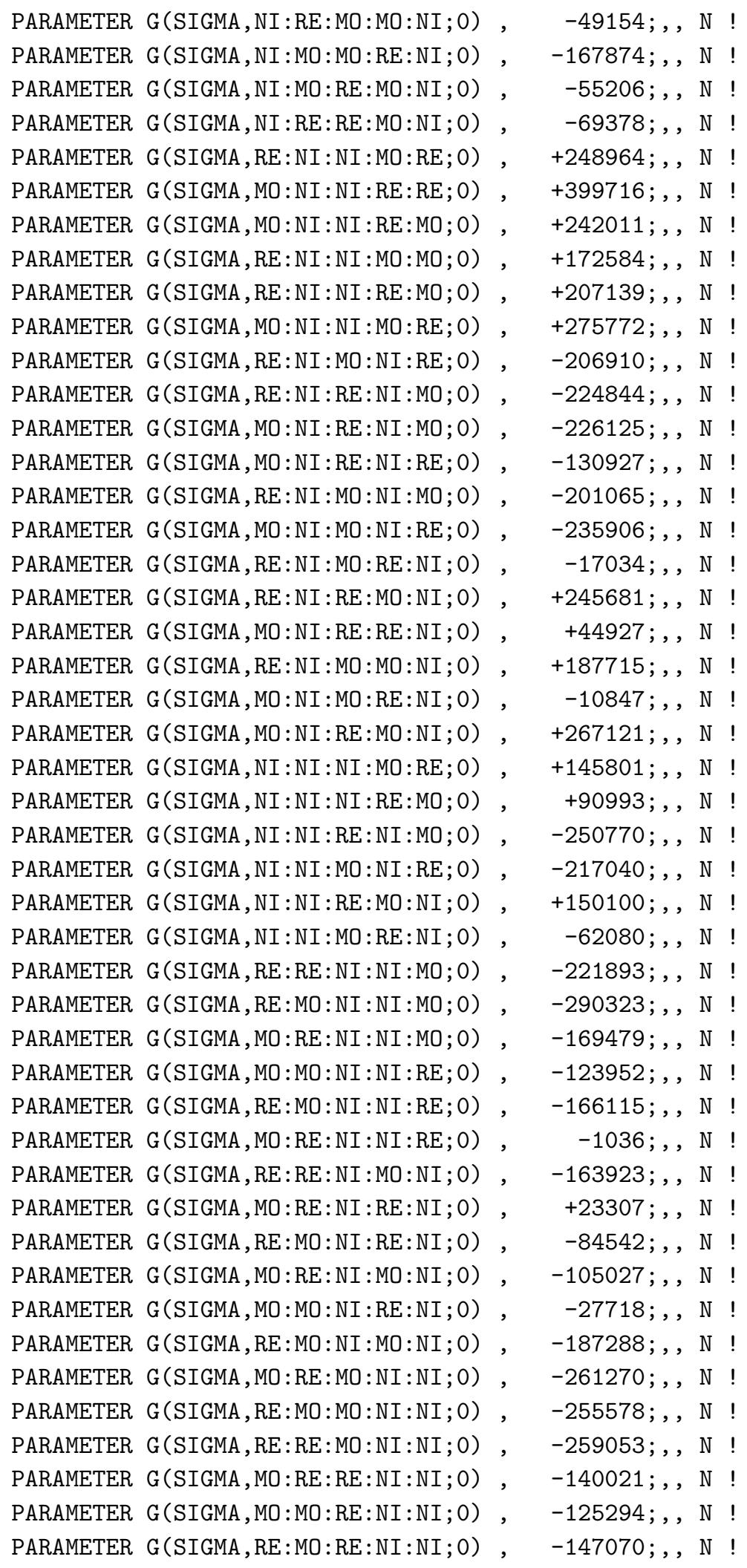




\begin{tabular}{|c|c|c|}
\hline PARAMETER G(SIGMA,NI:RE:NI:NI:MO;0) & & $-249307 ;,, \mathrm{N} !$ \\
\hline ARAMETER G(SIGMA, NI:MO:NI:NI:RE;0) & & $-195300 ;,, N$ \\
\hline AMETER G(SIGMA,NI:MO:NI:RE:NI;0) & & $-203914 ;,$, \\
\hline RAMETER G(SIGMA, NI:RE:NI:MO:NI;0) & & $-262456 ;,$ \\
\hline ARAMETER G(SIGMA, NI:RE:MO:NI:NI;0) & & $-296342 ;,$ \\
\hline ARAMETER G(SIGMA, NI:MO:RE:NI:NI;0) & & $-211810 ;,$ \\
\hline ARAMETER G(SIGMA,MO:NI:NI:NI:RE;0) & & $+38059 ;$ \\
\hline ARAMETER G(SIGMA, RE:NI:NI:NI:MO;0) & & $-147017 ;,$ \\
\hline PARAMETER G(SIGMA, MO:NI:NI:RE:NI;0) & & $+76990 ;,$ \\
\hline PARAMETER G(SIGMA, RE:NI:NI:MO:NI;0) & & $-58197 ;,$ \\
\hline PARAMETER G(SIGMA, RE:NI:MO:NI:NI;0) & & $-274143 ;,$ \\
\hline PARAMETER G(SIGMA, MO:NI:RE:NI:NI;0) & & $-147336 ;$ \\
\hline PARAMETER G(SIGMA, MO:RE:NI:NI:NI;0) & & -142540 \\
\hline PARAMETER G(SIGMA, RE:MO:NI:NI:NI;0) & & $-273243 ;,$ \\
\hline
\end{tabular}

\subsubsection{EBEF}

The functions used in the previous section are directly used as parameters in the EBEF. This description can currently only be used by the OpenCalphad [3] software. (See file MoNiRe_BEF.TDB in the supplemental materials.)

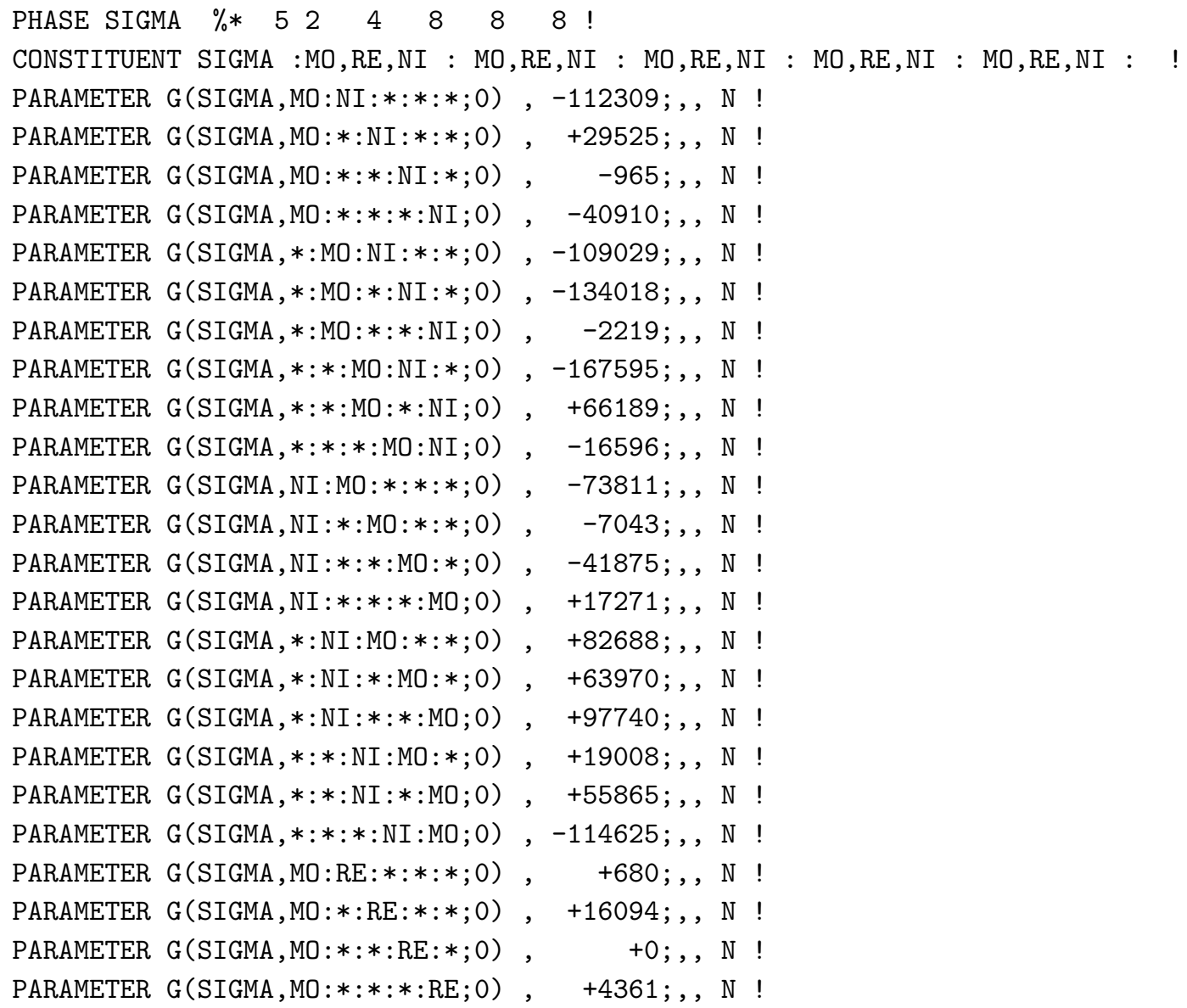




\begin{tabular}{|c|c|}
\hline RAMETER G(SIGMA, $*:$ MO :RE $: *: * ; 0)$ & $-14434 ;,, N$ \\
\hline RAMETER G(SIGMA, $*:$ MO $: *:$ RE $: * ; 0)$ & $-81839 ;,$, \\
\hline IGMA $, *: \mathrm{MO}: *: *: \mathrm{RE} ; 0)$ & $-28743 ;,$ \\
\hline GMA $, *: *: \mathrm{MO}: \mathrm{RE}: * ; 0)$ & -86 \\
\hline $\mathrm{MMA}, *: *: \mathrm{MO}: *: \mathrm{RE} ; 0)$ & -73 \\
\hline METER G(SIGMA, $*: *: *:$ MO $: \mathrm{RE} ; 0)$ & +21 \\
\hline AMETER G(SIGMA, RE:MO: $*: *: * ; 0)$ & -22 \\
\hline (SIGMA , RE: $*:$ MO $: *: * ; 0)$ & -15 \\
\hline $\mathrm{E}: *: *: \mathrm{MO}: * ; 0)$ & +5 \\
\hline SIGMA , RE: $*: *: *: \mathrm{MO} ; 0)$ & -30 \\
\hline $\mathrm{MA}, *: \mathrm{RE}: \mathrm{MO}: *: * ; 0)$ & +6 \\
\hline$(\mathrm{SIGMA}, *: \mathrm{RE}: *: \mathrm{MO}: * ; 0)$ & +42 \\
\hline (SIGMA $, *: \mathrm{RE}: *: *: \mathrm{MO} ; 0)$ & -25 \\
\hline , $*: *: \mathrm{RE}: \mathrm{MO}: * ; 0)$ & -1 \\
\hline$[\mathrm{GMA}, *: *: \mathrm{RE}$ & $3 ;,$ \\
\hline$(\mathrm{SIGMA}, *: *: *: \mathrm{RE}: \mathrm{MO} ; 0)$ & -119 \\
\hline$(\mathrm{SIGMA}, \mathrm{NI}: \mathrm{RE}: *: *: * ; 0)$ &,-1036 \\
\hline$[\mathrm{GMA}, \mathrm{NI}: *: \mathrm{RE}: *: * ; 0)$ & +206 \\
\hline$[\mathrm{I}: *: *: \mathrm{RE}: * ; 0)$ & -88 \\
\hline RAMETER G(SIGMA,NI $: *: *: *: \mathrm{RE} ; 0)$ & +327 \\
\hline$(\mathrm{SIGMA}, *: \mathrm{NI}: \mathrm{RE}: *: * ; 0)$ & -461 \\
\hline$(\mathrm{SIGMA}, *: \mathrm{NI}: *: \mathrm{RE}: * ; 0)$ & +91 \\
\hline$(\mathrm{SIGMA}, *: \mathrm{NI}: *: *: \mathrm{RE} ; 0)$ & +36 \\
\hline $\mathrm{MA}, *: *: \mathrm{NI}: \mathrm{RE}: * ; 0)$ & +70 \\
\hline$(\mathrm{SIGMA}, *: *: \mathrm{NI}: *: \mathrm{RE} ; 0)$ & , +136 \\
\hline $\mathrm{MA}, *: *: *: \mathrm{NI}: \mathrm{RE} ; 0)$ & -77 \\
\hline$E: N I: *: *: * ; 0)$ & -2 \\
\hline$(\mathrm{SIGMA}, \mathrm{RE}: *: \mathrm{NI}: *: * ; 0)$ & -19 \\
\hline RAMETER G(SIGMA, RE: $*: *:$ NI $: * ; 0)$ & -13 \\
\hline ARAMETER G(SIGMA, RE: $*: *: *: N I ; 0)$ & $-61558 ;$ \\
\hline ARAMETER G(SIGMA, $*:$ RE:NI: $*: * ; 0)$ & +5210 ; \\
\hline : $(\mathrm{SIGMA}, *: \mathrm{RE}: *: \mathrm{NI}: * ; 0)$ & $-89153 ;$ \\
\hline$(\mathrm{SIGMA}, *: \mathrm{RE}: *: *: \mathrm{NI} ; 0)$ & -2026 \\
\hline$(\mathrm{SIGMA}, *: *: \mathrm{RE}: \mathrm{NI}: * ; 0)$ & -89828 \\
\hline G(SIGMA $, *: *: \mathrm{RE}: *: \mathrm{NI} ; 0)$ & +75934 \\
\hline (SIGMA $, *: *: *: \mathrm{RE}: \mathrm{NI} ; 0)$ & $-24700 ;,$ \\
\hline
\end{tabular}

\subsubsection{CEF using the effective bond energies functions, identical to EBEF}

The functions listed here correspond to the bond parameters given in Table 2. They have been fitted for each binary system to minimize the difference between the end members of the 5SL model and the DFT results for the binary compounds. The terminology "AnBm" stands for the contribution considering A in the $n^{\text {th }}$ sublattice and B in the $m^{\text {th }}$ sublattice. The 90 binary and 150 ternary parameters of the CEF are then expressed with these functions.

This description is exactly identical to the one given in Sec. 5.2.3 and can be used by OpenCalphad [3], Thermo-Calc [33], or other software supporting the TDB format. The description in Sec. 5.2.3 can currently only be used by the OpenCalphad [3] software. (See file MoNiRe_CEF_BEF.TDB in the supplemental 


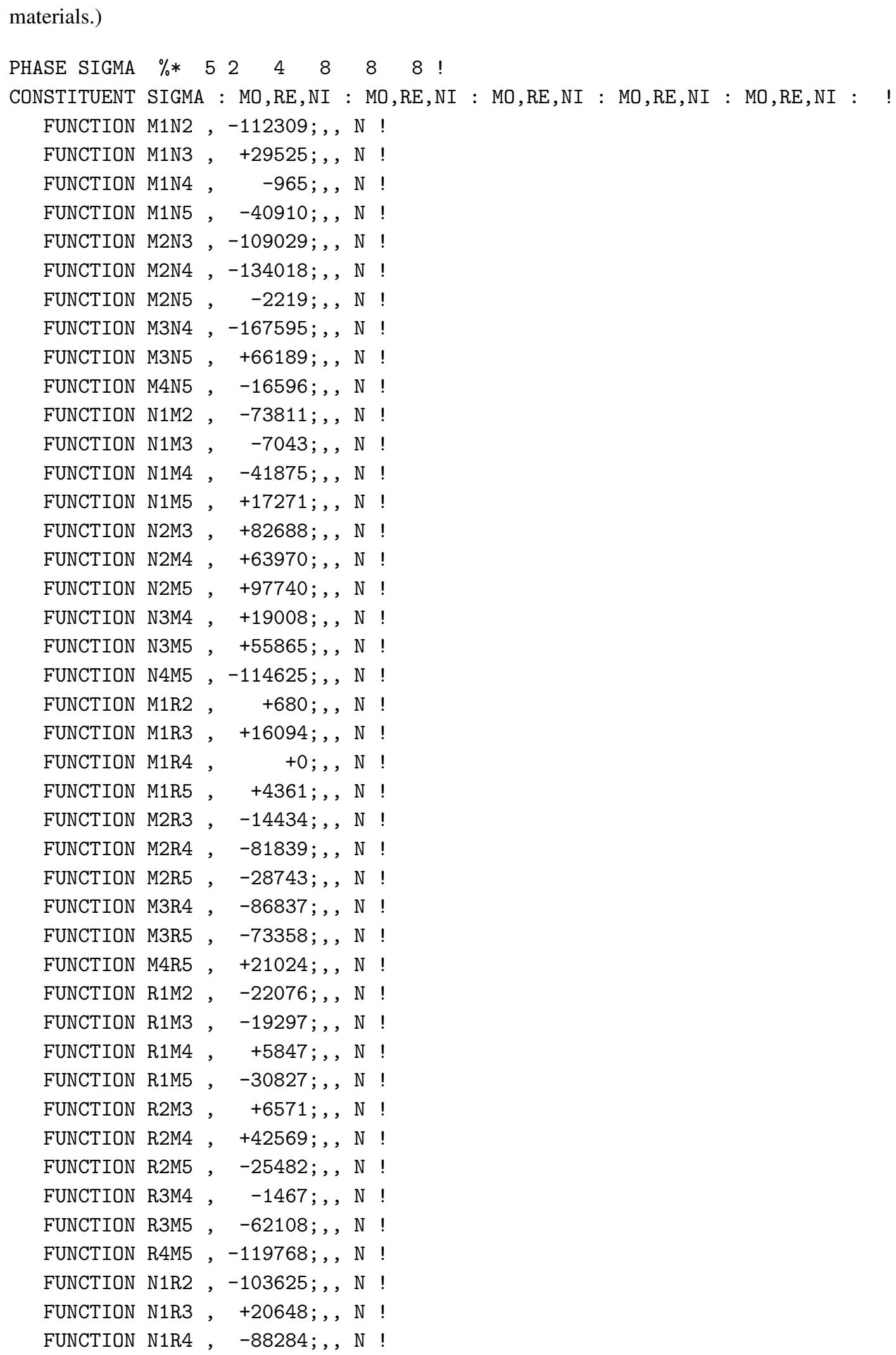




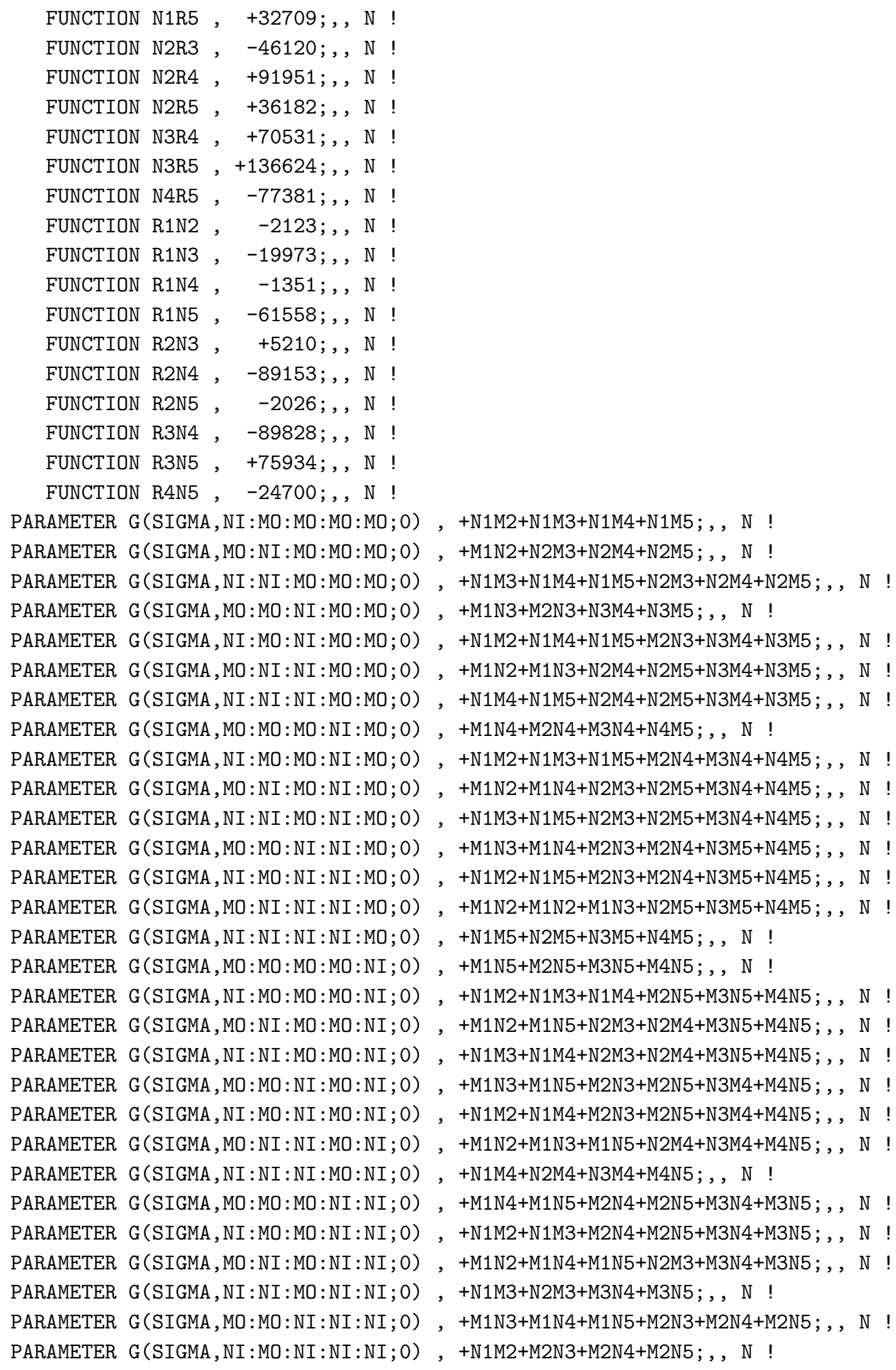


PARAMETER G(SIGMA, MO:NI:NI:NI:NI;0) , +M1N2+M1N3+M1N4+M1N5;,, N ! PARAMETER G(SIGMA,RE:MO:MO:MO:MO;0) , +R1M2+R1M3+R1M4+R1M5;, , N ! PARAMETER G(SIGMA,MO:RE:MO:MO:MO;0), +M1R2+R2M3+R2M4+R2M5;,, N ! PARAMETER G(SIGMA, RE:RE:MO:MO:MO;0) , +R1M3+R1M4+R1M5+R2M3+R2M4+R2M5; , N I PARAMETER G (SIGMA, MO:MO:RE:MO:MO;0), +M1R3+M2R3+R3M4+R3M5;, , N ! PARAMETER G(SIGMA, RE:MO:RE:MO:MO;0) , +R1M2+R1M4+R1M5+M2R3+R3M4+R3M5; , N I PARAMETER G(SIGMA,MO:RE:RE:MO:MO;0), +M1R2+M1R3+R2M4+R2M5+R3M4+R3M5;,, N ! PARAMETER G(SIGMA, RE:RE:RE:MO:MO;0) , +R1M4+R1M5+R2M4+R2M5+R3M4+R3M5; , N I PARAMETER G(SIGMA,MO:MO:MO:RE:MO;0) , +M1R4+M2R4+M3R4+R4M5;,, N ! PARAMETER G(SIGMA, RE:MO:MO:RE:MO;0) , +R1M2+R1M3+R1M5+M2R4+M3R4+R4M5; , N I PARAMETER G(SIGMA,MO:RE:MO:RE:MO;0), +M1R2+M1R4+R2M3+R2M5+M3R4+R4M5; , N I PARAMETER G(SIGMA, RE:RE:MO:RE:MO;0) , +R1M3+R1M5+R2M3+R2M5+M3R4+R4M5; , N I PARAMETER G(SIGMA,MO:MO:RE:RE:MO;0), +M1R3+M1R4+M2R3+M2R4+R3M5+R4M5; , N I PARAMETER G(SIGMA, RE:MO:RE:RE:MO;0) , +R1M2+R1M5+M2R3+M2R4+R3M5+R4M5; , N I PARAMETER G(SIGMA,MO:RE:RE:RE:MO;0), +M1R2+M1R2+M1R3+R2M5+R3M5+R4M5;, , N ! PARAMETER G(SIGMA, RE:RE:RE:RE:MO;0) , +R1M5+R2M5+R3M5+R4M5; , N ! PARAMETER G(SIGMA,MO:MO:MO:MO:RE;0), +M1R5+M2R5+M3R5+M4R5;,, N ! PARAMETER G(SIGMA, RE:MO:MO:MO:RE;0) , +R1M2+R1M3+R1M4+M2R5+M3R5+M4R5; , , N ! PARAMETER G(SIGMA,MO:RE:MO:MO:RE;0), +M1R2+M1R5+R2M3+R2M4+M3R5+M4R5; , N N ! PARAMETER G(SIGMA, RE:RE:MO:MO:RE;0) , +R1M3+R1M4+R2M3+R2M4+M3R5+M4R5; , N N PARAMETER G(SIGMA,MO:MO:RE:MO:RE;0), +M1R3+M1R5+M2R3+M2R5+R3M4+M4R5; , , N ! PARAMETER G(SIGMA, RE:MO:RE:MO:RE;0) , +R1M2+R1M4+M2R3+M2R5+R3M4+M4R5; , , N ! PARAMETER G(SIGMA,MO:RE:RE:MO:RE;0) , +M1R2+M1R3+M1R5+R2M4+R3M4+M4R5; , , N ! PARAMETER G (SIGMA, RE:RE:RE:MO:RE;0) , +R1M4+R2M4+R3M4+M4R5; , , N ! PARAMETER G(SIGMA,MO:MO:MO:RE:RE;0), +M1R4+M1R5+M2R4+M2R5+M3R4+M3R5; , N N PARAMETER G(SIGMA, RE:MO:MO:RE:RE;0) , +R1M2+R1M3+M2R4+M2R5+M3R4+M3R5; , N I PARAMETER G(SIGMA,MO:RE:MO:RE:RE;0) , +M1R2+M1R4+M1R5+R2M3+M3R4+M3R5; , N I PARAMETER G(SIGMA,RE:RE:MO:RE:RE;0) , +R1M3+R2M3+M3R4+M3R5; , N ! PARAMETER G(SIGMA,MO:MO:RE:RE:RE;0) , +M1R3+M1R4+M1R5+M2R3+M2R4+M2R5; , N N ! PARAMETER G(SIGMA, RE:MO:RE:RE:RE;0) , +R1M2+M2R3+M2R4+M2R5; , N I PARAMETER G(SIGMA,MO:RE:RE:RE:RE;0) , +M1R2+M1R3+M1R4+M1R5;,, N ! PARAMETER G(SIGMA,NI:RE:RE:RE:RE;0) , +N1R2+N1R3+N1R4+N1R5;,, N ! PARAMETER G(SIGMA,RE:NI:RE:RE:RE;0), +R1N2+N2R3+N2R4+N2R5;, , N ! PARAMETER G(SIGMA,NI:NI:RE:RE:RE;0), +N1R3+N1R4+N1R5+N2R3+N2R4+N2R5; , N I PARAMETER G(SIGMA,RE:RE:NI:RE:RE;0), +R1N3+R2N3+N3R4+N3R5;, , N ! PARAMETER G(SIGMA,NI:RE:NI:RE:RE;0) , +N1R2+N1R4+N1R5+R2N3+N3R4+N3R5; , , N ! PARAMETER G(SIGMA, RE:NI:NI:RE:RE;0) , +R1N2+R1N3+N2R4+N2R5+N3R4+N3R5; , , N ! PARAMETER G(SIGMA, NI:NI:NI:RE:RE;0) , +N1R4+N1R5+N2R4+N2R5+N3R4+N3R5; , , N ! PARAMETER G(SIGMA, RE:RE:RE:NI:RE;0) , +R1N4+R2N4+R3N4+N4R5; , , N ! PARAMETER G(SIGMA, NI:RE:RE:NI:RE;0) , +N1R2+N1R3+N1R5+R2N4+R3N4+N4R5; , , N ! PARAMETER G(SIGMA, RE:NI:RE:NI:RE;0) , +R1N2+R1N4+N2R3+N2R5+R3N4+N4R5; , , N ! PARAMETER G(SIGMA,NI:NI:RE:NI:RE;0) , +N1R3+N1R5+N2R3+N2R5+R3N4+N4R5; , , N ! PARAMETER G(SIGMA, RE:RE:NI:NI:RE;0), +R1N3+R1N4+R2N3+R2N4+N3R5+N4R5;, , N ! PARAMETER G(SIGMA, NI:RE:NI:NI:RE;0), +N1R2+N1R5+R2N3+R2N4+N3R5+N4R5; , , N ! PARAMETER G(SIGMA, RE:NI:NI:NI:RE;0) , +R1N2+R1N2+R1N3+N2R5+N3R5+N4R5; , , N ! PARAMETER G(SIGMA,NI:NI:NI:NI:RE;0) , +N1R5+N2R5+N3R5+N4R5;,, N ! 
PARAMETER G(SIGMA,RE:RE:RE:RE:NI;0) , +R1N5+R2N5+R3N5+R4N5;, , N ! PARAMETER G(SIGMA,NI:RE:RE:RE:NI;0) , +N1R2+N1R3+N1R4+R2N5+R3N5+R4N5;,, N ! PARAMETER G(SIGMA, RE:NI:RE:RE:NI;0) , +R1N2+R1N5+N2R3+N2R4+R3N5+R4N5;,, N ! PARAMETER G(SIGMA,NI:NI:RE:RE:NI;0) , +N1R3+N1R4+N2R3+N2R4+R3N5+R4N5;,, N ! PARAMETER G(SIGMA, RE:RE:NI:RE:NI;0), +R1N3+R1N5+R2N3+R2N5+N3R4+R4N5;,, N ! PARAMETER G(SIGMA,NI:RE:NI:RE:NI;0) , +N1R2+N1R4+R2N3+R2N5+N3R4+R4N5;,, N ! PARAMETER G(SIGMA,RE:NI:NI:RE:NI;0) , +R1N2+R1N3+R1N5+N2R4+N3R4+R4N5;,, N ! PARAMETER G(SIGMA,NI:NI:NI:RE:NI;0), +N1R4+N2R4+N3R4+R4N5;, , N ! PARAMETER G(SIGMA, RE:RE:RE:NI:NI;0) , +R1N4+R1N5+R2N4+R2N5+R3N4+R3N5;,, N ! PARAMETER G(SIGMA,NI:RE:RE:NI:NI;0) , +N1R2+N1R3+R2N4+R2N5+R3N4+R3N5;,, N ! PARAMETER G(SIGMA, RE:NI:RE:NI:NI;0) , +R1N2+R1N4+R1N5+N2R3+R3N4+R3N5;,, N ! PARAMETER G(SIGMA,NI:NI:RE:NI:NI;0), +N1R3+N2R3+R3N4+R3N5;, , N ! PARAMETER G(SIGMA, RE:RE:NI:NI:NI;0) , +R1N3+R1N4+R1N5+R2N3+R2N4+R2N5;, , N ! PARAMETER G(SIGMA,NI:RE:NI:NI:NI;0), +N1R2+R2N3+R2N4+R2N5;, , N ! PARAMETER G(SIGMA,RE:NI:NI:NI:NI;0), +R1N2+R1N3+R1N4+R1N5;, , N ! PARAMETER G(SIGMA,MO:MO:MO:RE:NI;0) , M1R4+M1N5+M2R4+M2N5+M3R4+M3N5+R4N5;,, N ! PARAMETER G(SIGMA,MO:MO:RE:MO:NI;0) , M1R3+M1N5+M2R3+M2N5+R3M4+R3N5+M4N5;,, N ! PARAMETER G(SIGMA,MO:MO:RE:NI:MO;0) , M1R3+M1N4+M2R3+M2N4+R3N4+R3M5+N4M5;,, N ! PARAMETER G(SIGMA,MO:RE:MO:MO:NI;0) , M1R2+M1N5+R2M3+R2M4+R2N5+M3N5+M4N5;, N ! PARAMETER G(SIGMA,MO:RE:MO:NI:MO;0) , M1R2+M1N4+R2M3+R2N4+R2M5+M3N4+N4M5;,, N ! PARAMETER G(SIGMA,MO:RE:NI:MO:MO;0) , M1R2+M1N3+R2N3+R2M4+R2M5+N3M4+N3M5;,, N ! PARAMETER G(SIGMA,RE:MO:MO:MO:NI;0) , R1M2+R1M3+R1M4+R1N5+M2N5+M3N5+M4N5;,, N ! PARAMETER G(SIGMA,RE:MO:MO:NI:MO;0) , R1M2+R1M3+R1N4+R1M5+M2N4+M3N4+N4M5;,, N ! PARAMETER G(SIGMA,RE:MO:NI:MO:MO;0) , R1M2+R1N3+R1M4+R1M5+M2N3+N3M4+N3M5;,, N ! PARAMETER G(SIGMA,RE:NI:MO:MO:MO;0) , R1N2+R1M3+R1M4+R1M5+N2M3+N2M4+N2M5;,, N ! PARAMETER G(SIGMA,MO:MO:RE:RE:NI;0) , M1R3+M1R4+M1N5+M2R3+M2R4+M2N5+R3N5+R4N5;,, N ! PARAMETER G(SIGMA,MO:MO:RE:NI:RE;0) , M1R3+M1N4+M1R5+M2R3+M2N4+M2R5+R3N4+N4R5;,, N ! PARAMETER G(SIGMA,MO:MO:NI:RE:RE;0) , M1N3+M1R4+M1R5+M2N3+M2R4+M2R5+N3R4+N3R5;,, N ! PARAMETER G(SIGMA,MO:RE:MO:RE:NI;0) , M1R2+M1R4+M1N5+R2M3+R2N5+M3R4+M3N5+R4N5;,, N ! PARAMETER G(SIGMA,MO:RE:MO:NI:RE;0) , M1R2+M1N4+M1R5+R2M3+R2N4+M3N4+M3R5+N4R5;,, N ! PARAMETER G(SIGMA,MO:NI:MO:RE:RE;0) , M1N2+M1R4+M1R5+N2M3+N2R4+N2R5+M3R4+M3R5;,, N ! PARAMETER G(SIGMA,MO:RE:RE:MO:NI;0) , M1R2+M1R3+M1N5+R2M4+R2N5+R3M4+R3N5+M4N5;,, N ! PARAMETER G(SIGMA,MO:RE:NI:MO:RE;0) , M1R2+M1N3+M1R5+R2N3+R2M4+N3M4+N3R5+M4R5;,, N ! PARAMETER G(SIGMA,MO:NI:RE:MO:RE;0) , M1N2+M1R3+M1R5+N2R3+N2M4+N2R5+R3M4+M4R5;,, N ! PARAMETER G(SIGMA,MO:RE:RE:NI:MO;0) , M1R2+M1R3+M1N4+R2N4+R2M5+R3N4+R3M5+N4M5;,, N ! PARAMETER G(SIGMA,MO:RE:NI:RE:MO;0), M1R2+M1N3+M1R4+R2N3+R2M5+N3R4+N3M5+R4M5;, N ! PARAMETER G(SIGMA,MO:RE:NI:NI:MO;0) , M1R2+M1N3+M1N4+R2N3+R2N4+R2M5+N3M5+N4M5;,, N ! PARAMETER G(SIGMA, RE:MO:MO:NI:NI;0) , R1M2+R1M3+R1N4+R1N5+M2N4+M2N5+M3N4+M3N5;,, N ! PARAMETER G(SIGMA, RE:MO:NI:MO:NI;0) , R1M2+R1N3+R1M4+R1N5+M2N3+M2N5+N3M4+M4N5;,, N ! PARAMETER G(SIGMA,RE:MO:NI:NI:MO;0) , R1M2+R1N3+R1N4+R1M5+M2N3+M2N4+N3M5+N4M5;,, N ! PARAMETER G(SIGMA,MO:MO:MO:NI:RE;0) , M1N4+M1R5+M2N4+M2R5+M3N4+M3R5+N4R5;,, N ! PARAMETER G(SIGMA,MO:MO:NI:MO:RE;0) , M1N3+M1R5+M2N3+M2R5+N3M4+N3R5+M4R5;,, N ! PARAMETER G(SIGMA,MO:MO:NI:RE:MO;0), M1N3+M1R4+M2N3+M2R4+N3R4+N3M5+R4M5;,, N ! PARAMETER G(SIGMA,MO:NI:MO:MO:RE;0) , M1N2+M1R5+N2M3+N2M4+N2R5+M3R5+M4R5;,, N ! PARAMETER G(SIGMA,MO:NI:MO:RE:MO;0) , M1N2+M1R4+N2M3+N2R4+N2M5+M3R4+R4M5;,, N ! PARAMETER G(SIGMA,MO:NI:RE:MO:MO;0) , M1N2+M1R3+N2R3+N2M4+N2M5+R3M4+R3M5;,, N ! 
PARAMETER G(SIGMA,NI:MO:MO:MO:RE;0) , N1M2+N1M3+N1M4+N1R5+M2R5+M3R5+M4R5; , , N ! PARAMETER G(SIGMA,NI:MO:MO:RE:MO;0) , N1M2+N1M3+N1R4+N1M5+M2R4+M3R4+R4M5; , , N ! PARAMETER G(SIGMA,NI:MO:RE:MO:MO;0), N1M2+N1R3+N1M4+N1M5+M2R3+R3M4+R3M5; , , N ! PARAMETER G(SIGMA,NI:RE:MO:MO:MO;0) , N1R2+N1M3+N1M4+N1M5+R2M3+R2M4+R2M5; , , N ! PARAMETER G(SIGMA,MO:MO:NI:NI:RE;0), M1N3+M1N4+M1R5+M2N3+M2N4+M2R5+N3R5+N4R5; , , N ! PARAMETER G(SIGMA,MO:MO:NI:RE:NI;0), M1N3+M1R4+M1N5+M2N3+M2R4+M2N5+N3R4+R4N5; , , N ! PARAMETER G(SIGMA,MO:MO:RE:NI:NI;0) , M1R3+M1N4+M1N5+M2R3+M2N4+M2N5+R3N4+R3N5;, , N ! PARAMETER G(SIGMA,MO:NI:MO:NI:RE;0), M1N2+M1N4+M1R5+N2M3+N2R5+M3N4+M3R5+N4R5;, , N ! PARAMETER G(SIGMA,MO:NI:MO:RE:NI;0) , M1N2+M1R4+M1N5+N2M3+N2R4+M3R4+M3N5+R4N5;, , N ! PARAMETER G(SIGMA, MO:RE:MO:NI:NI;0), M1R2+M1N4+M1N5+R2M3+R2N4+R2N5+M3N4+M3N5; , , N ! PARAMETER G(SIGMA,MO:NI:NI:MO:RE;0), M1N2+M1N3+M1R5+N2M4+N2R5+N3M4+N3R5+M4R5; , , N ! PARAMETER G(SIGMA,MO:NI:RE:MO:NI;0), M1N2+M1R3+M1N5+N2R3+N2M4+R3M4+R3N5+M4N5; , , N ! PARAMETER G(SIGMA,MO:RE:NI:MO:NI;0), M1R2+M1N3+M1N5+R2N3+R2M4+R2N5+N3M4+M4N5; , , N ! PARAMETER G(SIGMA,MO:NI:NI:RE:MO;0), M1N2+M1N3+M1R4+N2R4+N2M5+N3R4+N3M5+R4M5; , , N ! PARAMETER G(SIGMA, MO:NI:RE:NI:MO;0) , M1N2+M1R3+M1N4+N2R3+N2M5+R3N4+R3M5+N4M5;, , N ! PARAMETER G(SIGMA,MO:NI:RE:RE:MO;0) , M1N2+M1R3+M1R4+N2R3+N2R4+N2M5+R3M5+R4M5; , , N ! PARAMETER G(SIGMA,NI:MO:MO:RE:RE;0), N1M2+N1M3+N1R4+N1R5+M2R4+M2R5+M3R4+M3R5;, , N ! PARAMETER G (SIGMA, NI:MO:RE:MO:RE;0), N1M2+N1R3+N1M4+N1R5+M2R3+M2R5+R3M4+M4R5;, , N ! PARAMETER G(SIGMA,NI:MO:RE:RE:MO;0), N1M2+N1R3+N1R4+N1M5+M2R3+M2R4+R3M5+R4M5; , , N ! PARAMETER G(SIGMA,NI:NI:NI:RE:MO;0) , N1R4+N1M5+N2R4+N2M5+N3R4+N3M5+R4M5; , , N ! PARAMETER G(SIGMA,NI:NI:RE:NI:MO;0) , N1R3+N1M5+N2R3+N2M5+R3N4+R3M5+N4M5; , , N ! PARAMETER G(SIGMA,NI:NI:RE:MO:NI;0) , N1R3+N1M4+N2R3+N2M4+R3M4+R3N5+M4N5; , , N ! PARAMETER G(SIGMA,NI:RE:NI:NI:MO;0) , N1R2+N1M5+R2N3+R2N4+R2M5+N3M5+N4M5; , , N ! PARAMETER G(SIGMA,NI:RE:NI:MO:NI;0) , N1R2+N1M4+R2N3+R2M4+R2N5+N3M4+M4N5; , , N ! PARAMETER G(SIGMA,NI:RE:MO:NI:NI;0) , N1R2+N1M3+R2M3+R2N4+R2N5+M3N4+M3N5; , , N ! PARAMETER G(SIGMA,RE:NI:NI:NI:MO;0) , R1N2+R1N3+R1N4+R1M5+N2M5+N3M5+N4M5; , , N ! PARAMETER G(SIGMA,RE:NI:NI:MO:NI;0) , R1N2+R1N3+R1M4+R1N5+N2M4+N3M4+M4N5; , , N ! PARAMETER G(SIGMA,RE:NI:MO:NI:NI;0) , R1N2+R1M3+R1N4+R1N5+N2M3+M3N4+M3N5; , , N ! PARAMETER G(SIGMA,RE:MO:NI:NI:NI;0) , R1M2+R1N3+R1N4+R1N5+M2N3+M2N4+M2N5; , , N ! PARAMETER G(SIGMA,NI:NI:RE:RE:MO;0) , N1R3+N1R4+N1M5+N2R3+N2R4+N2M5+R3M5+R4M5; , , N ! PARAMETER G(SIGMA, NI:NI:RE:MO:RE;0) , N1R3+N1M4+N1R5+N2R3+N2M4+N2R5+R3M4+M4R5;, , N ! PARAMETER G(SIGMA,NI:NI:MO:RE:RE;0), N1M3+N1R4+N1R5+N2M3+N2R4+N2R5+M3R4+M3R5; , N ! PARAMETER G(SIGMA,NI:RE:NI:RE:MO;0) , N1R2+N1R4+N1M5+R2N3+R2M5+N3R4+N3M5+R4M5; , , N ! PARAMETER G(SIGMA,NI:RE:NI:MO:RE;0), N1R2+N1M4+N1R5+R2N3+R2M4+N3M4+N3R5+M4R5;, , N ! PARAMETER G(SIGMA,NI:MO:NI:RE:RE;0), N1M2+N1R4+N1R5+M2N3+M2R4+M2R5+N3R4+N3R5; , , N ! PARAMETER G(SIGMA, NI:RE:RE:NI:MO;0) , N1R2+N1R3+N1M5+R2N4+R2M5+R3N4+R3M5+N4M5; , , N ! PARAMETER G(SIGMA,NI:RE:MO:NI:RE;0), N1R2+N1M3+N1R5+R2M3+R2N4+M3N4+M3R5+N4R5;, , N ! PARAMETER G(SIGMA,NI:MO:RE:NI:RE;0), N1M2+N1R3+N1R5+M2R3+M2N4+M2R5+R3N4+N4R5; , , N ! PARAMETER G(SIGMA, NI:RE:RE:MO:NI;0) , N1R2+N1R3+N1M4+R2M4+R2N5+R3M4+R3N5+M4N5; , , N ! PARAMETER G(SIGMA, NI:RE:MO:RE:NI;0) , N1R2+N1M3+N1R4+R2M3+R2N5+M3R4+M3N5+R4N5;, , N ! PARAMETER G(SIGMA,NI:RE:MO:MO:NI;0) , N1R2+N1M3+N1M4+R2M3+R2M4+R2N5+M3N5+M4N5;, , N ! PARAMETER G(SIGMA, RE:NI:NI:MO:MO;0) , R1N2+R1N3+R1M4+R1M5+N2M4+N2M5+N3M4+N3M5; , N PARAMETER G(SIGMA, RE:NI:MO:NI:MO;0) , R1N2+R1M3+R1N4+R1M5+N2M3+N2M5+M3N4+N4M5; , , N ! PARAMETER G(SIGMA, RE:NI:MO:MO:NI;0), R1N2+R1M3+R1M4+R1N5+N2M3+N2M4+M3N5+M4N5; , , N ! PARAMETER G(SIGMA,NI:NI:NI:MO:RE;0), N1M4+N1R5+N2M4+N2R5+N3M4+N3R5+M4R5; , , N ! PARAMETER G(SIGMA,NI:NI:MO:NI:RE;0) , N1M3+N1R5+N2M3+N2R5+M3N4+M3R5+N4R5; , , N ! 
PARAMETER G(SIGMA,NI:NI:MO:RE:NI;0) , N1M3+N1R4+N2M3+N2R4+M3R4+M3N5+R4N5; , , N ! PARAMETER G(SIGMA,NI:MO:NI:NI:RE;0), N1M2+N1R5+M2N3+M2N4+M2R5+N3R5+N4R5; , , N ! PARAMETER G(SIGMA,NI:MO:NI:RE:NI;0), N1M2+N1R4+M2N3+M2R4+M2N5+N3R4+R4N5; , , N ! PARAMETER G(SIGMA,NI:MO:RE:NI:NI;0), N1M2+N1R3+M2R3+M2N4+M2N5+R3N4+R3N5;, , N ! PARAMETER G(SIGMA,MO:NI:NI:NI:RE;0) , M1N2+M1N3+M1N4+M1R5+N2R5+N3R5+N4R5; , , N ! PARAMETER G(SIGMA,MO:NI:NI:RE:NI;0) , M1N2+M1N3+M1R4+M1N5+N2R4+N3R4+R4N5; , , N ! PARAMETER G(SIGMA,MO:NI:RE:NI:NI;0) , M1N2+M1R3+M1N4+M1N5+N2R3+R3N4+R3N5; , , N ! PARAMETER G(SIGMA,MO:RE:NI:NI:NI;0) , M1R2+M1N3+M1N4+M1N5+R2N3+R2N4+R2N5; , , N ! PARAMETER G(SIGMA,NI:NI:MO:MO:RE;0), N1M3+N1M4+N1R5+N2M3+N2M4+N2R5+M3R5+M4R5; , , N ! PARAMETER G(SIGMA,NI:NI:MO:RE:MO;0), N1M3+N1R4+N1M5+N2M3+N2R4+N2M5+M3R4+R4M5; , , N ! PARAMETER G(SIGMA,NI:NI:RE:MO:MO;0), N1R3+N1M4+N1M5+N2R3+N2M4+N2M5+R3M4+R3M5; , , N ! PARAMETER G(SIGMA,NI:MO:NI:MO:RE;0), N1M2+N1M4+N1R5+M2N3+M2R5+N3M4+N3R5+M4R5; , , N ! PARAMETER G(SIGMA,NI:MO:NI:RE:MO;0), N1M2+N1R4+N1M5+M2N3+M2R4+N3R4+N3M5+R4M5; , , N ! PARAMETER G(SIGMA,NI:RE:NI:MO:MO;0), N1R2+N1M4+N1M5+R2N3+R2M4+R2M5+N3M4+N3M5; , , N ! PARAMETER G(SIGMA,NI:MO:MO:NI:RE;0) , N1M2+N1M3+N1R5+M2N4+M2R5+M3N4+M3R5+N4R5;, , N ! PARAMETER G(SIGMA,NI:MO:RE:NI:MO;0) , N1M2+N1R3+N1M5+M2R3+M2N4+R3N4+R3M5+N4M5; , , N ! PARAMETER G(SIGMA,NI:RE:MO:NI:MO;0) , N1R2+N1M3+N1M5+R2M3+R2N4+R2M5+M3N4+N4M5; , , N ! PARAMETER G(SIGMA,NI:MO:MO:RE:NI;0), N1M2+N1M3+N1R4+M2R4+M2N5+M3R4+M3N5+R4N5;, , N ! PARAMETER G(SIGMA,NI:MO:RE:MO:NI;0), N1M2+N1R3+N1M4+M2R3+M2N5+R3M4+R3N5+M4N5; , , N ! PARAMETER G(SIGMA,NI:MO:RE:RE:NI;0), N1M2+N1R3+N1R4+M2R3+M2R4+M2N5+R3N5+R4N5; , , N ! PARAMETER G(SIGMA,MO:NI:NI:RE:RE;0), M1N2+M1N3+M1R4+M1R5+N2R4+N2R5+N3R4+N3R5; , , N ! PARAMETER G(SIGMA, MO:NI:RE:NI:RE;0) , M1N2+M1R3+M1N4+M1R5+N2R3+N2R5+R3N4+N4R5; , , N ! PARAMETER G(SIGMA, MO:NI:RE:RE:NI;0), M1N2+M1R3+M1R4+M1N5+N2R3+N2R4+R3N5+R4N5; , , N ! PARAMETER G(SIGMA,RE:RE:RE:MO:NI;0) , R1M4+R1N5+R2M4+R2N5+R3M4+R3N5+M4N5; , , N ! PARAMETER G(SIGMA,RE:RE:MO:RE:NI;0) , R1M3+R1N5+R2M3+R2N5+M3R4+M3N5+R4N5; , , N ! PARAMETER G(SIGMA,RE:RE:MO:NI:RE;0) , R1M3+R1N4+R2M3+R2N4+M3N4+M3R5+N4R5; , , N ! PARAMETER G(SIGMA,RE:MO:RE:RE:NI;0) , R1M2+R1N5+M2R3+M2R4+M2N5+R3N5+R4N5; , , N ! PARAMETER G(SIGMA,RE:MO:RE:NI:RE;0) , R1M2+R1N4+M2R3+M2N4+M2R5+R3N4+N4R5; , , N ! PARAMETER G(SIGMA,RE:MO:NI:RE:RE;0) , R1M2+R1N3+M2N3+M2R4+M2R5+N3R4+N3R5; , , N ! PARAMETER G(SIGMA,MO:RE:RE:RE:NI;0) , M1R2+M1R3+M1R4+M1N5+R2N5+R3N5+R4N5; , , N ! PARAMETER G(SIGMA,MO:RE:RE:NI:RE;0) , M1R2+M1R3+M1N4+M1R5+R2N4+R3N4+N4R5; , , N ! PARAMETER G(SIGMA,MO:RE:NI:RE:RE;0) , M1R2+M1N3+M1R4+M1R5+R2N3+N3R4+N3R5; , , N ! PARAMETER G(SIGMA,MO:NI:RE:RE:RE;0) , M1N2+M1R3+M1R4+M1R5+N2R3+N2R4+N2R5; , , N ! PARAMETER G(SIGMA, RE:RE:MO:MO:NI;0) , R1M3+R1M4+R1N5+R2M3+R2M4+R2N5+M3N5+M4N5; , N $N$ PARAMETER G(SIGMA, RE:RE:MO:NI:MO;0) , R1M3+R1N4+R1M5+R2M3+R2N4+R2M5+M3N4+N4M5; , , N ! PARAMETER G(SIGMA, RE:RE:NI:MO:MO;0) , R1N3+R1M4+R1M5+R2N3+R2M4+R2M5+N3M4+N3M5; , , N ! PARAMETER G(SIGMA, RE:MO:RE:MO:NI;0) , R1M2+R1M4+R1N5+M2R3+M2N5+R3M4+R3N5+M4N5; , , N ! PARAMETER G(SIGMA,RE:MO:RE:NI:MO;0) , R1M2+R1N4+R1M5+M2R3+M2N4+R3N4+R3M5+N4M5; , , N ! PARAMETER G(SIGMA, RE:NI:RE:MO:MO;0) , R1N2+R1M4+R1M5+N2R3+N2M4+N2M5+R3M4+R3M5; , , N ! PARAMETER G (SIGMA, RE:MO:MO:RE:NI;0) , R1M2+R1M3+R1N5+M2R4+M2N5+M3R4+M3N5+R4N5; , , N ! PARAMETER G(SIGMA, RE:MO:NI:RE:MO;0) , R1M2+R1N3+R1M5+M2N3+M2R4+N3R4+N3M5+R4M5; , , N ! PARAMETER G(SIGMA,RE:NI:MO:RE:MO;0) , R1N2+R1M3+R1M5+N2M3+N2R4+N2M5+M3R4+R4M5;, , N ! PARAMETER G(SIGMA, RE:MO:MO:NI:RE;0) , R1M2+R1M3+R1N4+M2N4+M2R5+M3N4+M3R5+N4R5; , , N ! PARAMETER G(SIGMA, RE:MO:NI:MO:RE;0), R1M2+R1N3+R1M4+M2N3+M2R5+N3M4+N3R5+M4R5; , , N ! PARAMETER G(SIGMA, RE:MO:NI:NI:RE;0) , R1M2+R1N3+R1N4+M2N3+M2N4+M2R5+N3R5+N4R5; , , N ! PARAMETER G(SIGMA, MO:RE:RE:NI:NI;0) , M1R2+M1R3+M1N4+M1N5+R2N4+R2N5+R3N4+R3N5; , , N ! 


\begin{abstract}
PARAMETER G(SIGMA,MO:RE:NI:RE:NI;0) , M1R2+M1N3+M1R4+M1N5+R2N3+R2N5+N3R4+R4N5;,, N ! PARAMETER G(SIGMA,MO:RE:NI:NI:RE;0) , M1R2+M1N3+M1N4+M1R5+R2N3+R2N4+N3R5+N4R5;,, N ! PARAMETER G(SIGMA,RE:RE:RE:NI:MO;0) , R1N4+R1M5+R2N4+R2M5+R3N4+R3M5+N4M5;, N ! PARAMETER G(SIGMA,RE:RE:NI:RE:MO;0) , R1N3+R1M5+R2N3+R2M5+N3R4+N3M5+R4M5;, N ! PARAMETER G(SIGMA,RE:RE:NI:MO:RE;0) , R1N3+R1M4+R2N3+R2M4+N3M4+N3R5+M4R5;,, N ! PARAMETER G(SIGMA,RE:NI:RE:RE:MO;0) , R1N2+R1M5+N2R3+N2R4+N2M5+R3M5+R4M5;,, N ! PARAMETER G(SIGMA,RE:NI:RE:MO:RE;0) , R1N2+R1M4+N2R3+N2M4+N2R5+R3M4+M4R5;,, N ! PARAMETER G(SIGMA,RE:NI:MO:RE:RE;0) , R1N2+R1M3+N2M3+N2R4+N2R5+M3R4+M3R5;,, N ! PARAMETER G(SIGMA,NI:RE:RE:RE:MO;0) , N1R2+N1R3+N1R4+N1M5+R2M5+R3M5+R4M5;,, N ! PARAMETER G(SIGMA,NI:RE:RE:MO:RE;0) , N1R2+N1R3+N1M4+N1R5+R2M4+R3M4+M4R5;,, N ! PARAMETER G(SIGMA,NI:RE:MO:RE:RE;0) , N1R2+N1M3+N1R4+N1R5+R2M3+M3R4+M3R5;,, N ! PARAMETER G(SIGMA, NI:MO:RE:RE:RE;0) , N1M2+N1R3+N1R4+N1R5+M2R3+M2R4+M2R5;,, N ! PARAMETER G(SIGMA, RE:RE:NI:NI:MO;0) , R1N3+R1N4+R1M5+R2N3+R2N4+R2M5+N3M5+N4M5;,, N ! PARAMETER G(SIGMA,RE:RE:NI:MO:NI;0) , R1N3+R1M4+R1N5+R2N3+R2M4+R2N5+N3M4+M4N5;, N ! PARAMETER G(SIGMA,RE:RE:MO:NI:NI;0) , R1M3+R1N4+R1N5+R2M3+R2N4+R2N5+M3N4+M3N5;,, N ! PARAMETER G(SIGMA, RE:NI:RE:NI:MO;0) , R1N2+R1N4+R1M5+N2R3+N2M5+R3N4+R3M5+N4M5;,, N ! PARAMETER G(SIGMA, RE:NI:RE:MO:NI;0) , R1N2+R1M4+R1N5+N2R3+N2M4+R3M4+R3N5+M4N5;,, N ! PARAMETER G(SIGMA, RE:MO:RE:NI:NI;0) , R1M2+R1N4+R1N5+M2R3+M2N4+M2N5+R3N4+R3N5;,, N ! PARAMETER G(SIGMA,RE:NI:NI:RE:MO;0) , R1N2+R1N3+R1M5+N2R4+N2M5+N3R4+N3M5+R4M5;,, N ! PARAMETER G(SIGMA, RE:NI:MO:RE:NI;0) , R1N2+R1M3+R1N5+N2M3+N2R4+M3R4+M3N5+R4N5;,, N ! PARAMETER G(SIGMA,RE:MO:NI:RE:NI;0) , R1M2+R1N3+R1N5+M2N3+M2R4+M2N5+N3R4+R4N5;,, N ! PARAMETER G(SIGMA, RE:NI:NI:MO:RE;0) , R1N2+R1N3+R1M4+N2M4+N2R5+N3M4+N3R5+M4R5;,, N ! PARAMETER G(SIGMA, RE:NI:MO:NI:RE;0) , R1N2+R1M3+R1N4+N2M3+N2R5+M3N4+M3R5+N4R5;,, N ! PARAMETER G(SIGMA,RE:NI:MO:MO:RE;0) , R1N2+R1M3+R1M4+N2M3+N2M4+N2R5+M3R5+M4R5;, N ! PARAMETER G(SIGMA, NI:RE:RE:MO:MO;0) , N1R2+N1R3+N1M4+N1M5+R2M4+R2M5+R3M4+R3M5;,, N ! PARAMETER G(SIGMA, NI:RE:MO:RE:MO;0) , N1R2+N1M3+N1R4+N1M5+R2M3+R2M5+M3R4+R4M5;,, N ! PARAMETER G(SIGMA,NI:RE:MO:MO:RE;0) , N1R2+N1M3+N1M4+N1R5+R2M3+R2M4+M3R5+M4R5;,, N !
\end{abstract}

\title{
Supplemental Materials
}

Supplemental materials include text files with thermodynamic descriptions in TDB format for Al-Cr-Ni and Mo-Ni-Re using the compound energy formalism (CEF), effective bond energy formalism in compound energy formalism notation (CEF_BEF), and the effective bond energy formalism (BEF). The notation 2SL and 4 SL indicates the number of sublattices used to describe fcc ordering. Whether only binary or binary and ternary parameters were used for the $\sigma$ phase is indicated by the letters $b$ and $t$, respectively.

- AlCrNi_CEF_2SL.TDB

- AlCrNi_CEF_4SL.TDB

- AlCrNi_CEF_4SLF.TDB

- AlCrNi_BEF.TDB

- MoNiRe_CEF_b.TDB

- MoNiRe_CEF_t.TDB

- MoNiRe_CEF_BEF.TDB

- MoNiRe_BEF.TDB 


\section{Acknowledgments}

SGF acknowledges funding by the Deutsche Forschungsgemeinschaft (DFG) through project C6 of the collaborative research centre SFB/TR 103.

\section{References}

[1] Saunders N, Miodownik AP (1998) CALPHAD (Calculation of Phase Diagrams): A Comprehensive Guide (Pergamon Press, Elsevier Science, Oxford, UK).

[2] Lukas HL, Fries SG, Sundman B (2007) Computational Thermodynamics: The Calphad Method (Cambridge University Press, Cambridge, UK).

[3] OpenCalphad. Available at https://github.com/sundmanbo/opencalphad.

[4] Andersson J-O, Fernández Guillermet A, Hillert M, Jansson B, Sundman B (1986) A compound-energy model of ordering in a phase with sites of different coordination numbers. Acta Metallurgica 34:437-445. https://doi.org/10.1016/0001-6160(86)90079-9

[5] Hillert M (2001) The compound energy formalism. Journal of Alloys and Compounds 320:161-176. https://doi.org/10.1016/S0925-8388(00)01481-X

[6] Ansara I, Chart TG, Fernández Guillermet A, Hayes FH, Kattner UR, Pettifor DG, Saunders N, Zeng K (1997) Thermodynamic modelling of selected topologically close-packed intermetallic compounds. Calphad 21(2):171-218. https://doi.org/10.1016/S0364-5916(97)00021-7

[7] Joubert J-M (2008) Crystal chemistry and Calphad modeling of the $\sigma$ phase. Progress in Materials Science 53:528-583. https://doi.org/10.1016/j.pmatsci.2007.04.001

[8] Andersson J-O, Sundman B (1987) Thermodynamic properties of the Cr-Fe system. Calphad 11:83-92. https://doi.org/10.1016/0364-5916(87)90021-6

[9] Ansara I, Burton B, Chen Q, Hillert M, Fernández Guillermet A, Fries SG, Lukas HL, Seifert HJ, Oates WA (2000) Models for composition dependence. Calphad 24(1):19-40. https://doi.org/10.1016/S0364-5916(00)00013-4

[10] Oates WA, Spencer PJ, Fries SG (1996) A cluster expansion for Cu-Au alloys based on experimental data. Calphad 20(4):481-489. https://doi.org/10.1016/S0364-5916(97)00010-2

[11] Dupin N, Sundman B (2001) A thermodynamic database for Ni-base superalloys. Scandinavian Journal of Metallurgy 30(3):184-192. https://doi.org/10.1034/j.1600-0692.2001.300309.x

[12] Dupin N (2004) Thermodynamic database for Ni-base alloys. Unpublished research.

[13] Hallstedt B, Dupin N, Hillert M, Höglund L, Lukas HL, Schuster JC, Solak N (2007) Thermodynamic models for crystalline phases. Composition dependent models for volume, bulk modulus and thermal expansion. Calphad 31(1):28-37. https://doi.org/10.1016/j.calphad.2006.02.008

[14] Fries SG, Sundman B (2002) Using Re-W $\sigma$-phase first-principles results in the Bragg-Williams approximation to calculate finite-temperature thermodynamic properties. Physical Review B 66:012203. https://doi.org/10.1103/PhysRevB.66.012203

[15] Berne C, Sluiter M, Kawazoe Y, Hansen T, Pasturel A (2001) Site occupancy in the Re-W $\sigma$ phase. Physical Review B 64:144103. https://doi.org/10.1103/PhysRevB.64.144103

[16] Palumbo M, Abe T, Kocer C, Murakami H, Onodera H (2010) Ab initio and thermodynamic study of the Cr-Re system. Calphad 34:495-503. https://doi.org/10.1016/j.calphad.2010.09.003

[17] Mathieu R, Dupin N, Crivello J-C, Yaqoob K, Breidi A, Fiorani JM, David N, Joubert J-M (2013) Calphad description of the Mo-Re system focused on the $\sigma$ phase modeling. Calphad 43:18-31. https://doi.org/10.1016/j.calphad.2013.08.002

[18] Li Z, Mao H, Korzhavyi P, Selleby M (2016) Thermodynamic re-assessment of the Co-Cr system supported by first-principles calculations. Calphad 52:1-7. https://doi.org/0.1016/j.calphad.2015.10.013

[19] Korzhavyi P, Sundman B, Selleby M, Johansson B (2005) Atomic, electronic, and magnetic structure of iron-based $\sigma$-phases. Materials Research Society Symposium Proceedings 842:517-522.

[20] Frisk K (1990) A thermodynamic evaluation of the Mo-Ni system. Calphad 14(3):311-320. https://doi.org/10.1016/0364-5916(90)90031-T

[21] Yaqoob K, Joubert J-M (2012) Experimental determination and thermodynamic modeling of the Ni-Re binary system. Journal of Solid State Chemistry 196:320-325. https://doi.org/10.1016/j.jssc.2012.06.036

[22] Yaqoob K, Crivello J-C, Joubert J-M (2012) Comparison of the site occupancies determined by combined Rietveld refinement and density functional theory calculations: Example of the ternary Mo-Ni-Re $\sigma$ phase. Inorganic Chemistry 51(5):3071-3078 https://doi.org/10.1021/ic202479y

[23] Kodentsov AA, Dunae SF, Slyusarenko EM, Sokolovskaya EM, Priimak AN (1987) Phase equilibria in the rhenium-molybdenum-nickel system. Vestnik Moskovskogo Universiteta Ser2 Khimiya 28:153-158.

[24] Borisov VA, Yaschenko AI, Slyusarenko EM, Dunaev SF (1992) An investigation of solid-phase interactions in the Ni-Mo-Re system at 1425 K. Moscow University Chemistry Bulletin 47:76-79. 
[25] Slyusarenko EM, Peristyi AV, Kerimov EY, Sofin MV, Skorbov DYu (1998) Ternary systems of nickel and rhenium with transition metals. Journal of Alloys and Compounds 264:180-189. https://doi.org/10.1016/S0925-8388(97)00238-7

[26] Feng Y, Wang R, Yu K, Wen D (2008) Determination of isothermal sections of the Co-Nb-Ni and Ni-Mo-Re ternary systems. Rare Metals 27:83-88. https://doi.org/10.1016/S1001-0521(08)60036-4

[27] Yaqoob K, Joubert J-M (2013) Experimental investigation of the Mo-Ni-Re system. Journal of Alloys and Compounds 559:101-111. https://doi.org/10.1016/j.jallcom.2013.01.045

[28] Dupin N, Ansara I, Sundman B (2001) Thermodynamic re-assessment of the ternary system Al-Cr-Ni. Calphad 25(2):279-298. https://doi.org/10.1016/S0364-5916(01)00049-9

[29] Kusoffsky A, Dupin N, Sundman B (2001) On the compound energy formalism applied to fcc ordering. Calphad 25(4):549-565. https://doi.org/10.1016/S0364-5916(02)00007-X

[30] Shockley W (1938) Theory of order for the copper gold alloy system. Journal of Chemical Physics 6:130-144. https://doi.org/10.1063/1.1750214

[31] Sluiter MHF, Kawazoe Y (1995) Site preference of ternary additions to ni 3 al. Physical Review B 51(7):4062-4073. https://doi.org/10.1103/PhysRevB.51.4062

[32] Oates WA, Wenzl H (1993) Bond energy model of multiple sublattice solutions using species chemical potentials. basic theory and relation to compound energy model. Calphad 17(1):35-46. https://doi.org/10.1016/0364-5916(93)90032-7

[33] Thermo-Calc Software. Available at http://www.thermocalc.com/.

About the authors: Nathalie Dupin is a self-employed researcher at Calcul Thermodynamique, France. She develops Calphad-type thermodynamic databases and models for academic and commercial customers.

Ursula Kattner is a physical scientist in the Thermodynamics and Kinetics Group of the Materials Science and Engineering Division of the Material Measurement Laboratory at NIST. She carries out research in computational thermodynamics with a focus on the application to material and process development.

Bo Sundman is a professor emeritus in the Computational Thermodynamics Division of the Materials Science and Engineering Department of the Royal Institute of Technology, Sweden. He carries out research in computational thermodynamics, software, database, and model development.

Mauro Palumbo is a self-employed researcher at COMPUMAT, Italy. His research focuses on providing first-principles data for the development of thermodynamic databases.

Suzana Fries is the leader of the Computational Thermodynamics/Calphad Group of the Scalebridging Thermodynamic and Kinetic Simulation Department of the Interdisciplinary Centre for Advanced Materials Simulation at Ruhr-Universität Bochum, Germany. Her research focuses on the application and development of Calphad databases and the implementation of results from first-principles calculations.

The National Institute of Standards and Technology is an agency of the U.S. Department of Commerce. 